\title{
Maximum common property: a new approach for molecular similarity
}

\author{
Aurelio Antelo-Collado ${ }^{\circledR 0}$, Ramón Carrasco-Velar ${ }^{1 *}$ [D, Nicolás García-Pedrajas ${ }^{2}$ [D \\ and Gonzalo Cerruela-Garcíaº
}

\begin{abstract}
The maximum common property similarity (MCPhd) method is presented using descriptors as a new approach to determine the similarity between two chemical compounds or molecular graphs. This method uses the concept of maximum common property arising from the concept of maximum common substructure and is based on the electrotopographic state index for atoms. A new algorithm to quantify the similarity values of chemical structures based on the presented maximum common property concept is also developed in this paper. To verify the validity of this approach, the similarity of a sample of compounds with antimalarial activity is calculated and compared with the results obtained by four different similarity methods: the small molecule subgraph detector (SMSD), molecular fingerprint based (OBabel_FP2), ISIDA descriptors and shape-feature similarity (SHAFTS). The results obtained by the MCPhd method differ significantly from those obtained by the compared methods, improving the quantification of the similarity. A major advantage of the proposed method is that it helps to understand the analogy or proximity between physicochemical properties of the molecular fragments or subgraphs compared with the biological response or biological activity. In this new approach, more than one property can be potentially used. The method can be considered a hybrid procedure because it combines descriptor and the fragment approaches.
\end{abstract}

Keywords: Maximum common property, Electrotopographic state index, Molecular similarity, Tanimoto function, Maximum common structure

\section{Introduction}

Molecular similarity is one of the most explored and employed concepts in cheminformatics (chemical informatics or chemoinformatics) [1]. Moreover, it is currently one of the central subjects in medicinal chemistry research $[1,2]$. Molecular similarity can be evaluated using different approaches, which can be classified into two principal categories: those based on descriptors and those based on substructures [3]. To estimate similarity among molecules, it is necessary to identify those

*Correspondence: rcarrasco@uci.cu

${ }^{1}$ University of Informatics Science, Carretera San Antonio de los Baños Km. 2 1/2, Boyeros, La Habana, Cuba, Havana, Cuba

Full list of author information is available at the end of the article structural or chemical/physical properties that are useful to correlate and then predict the relationships among them.

Similarity calculations based on molecular descriptors use fingerprint representations [3, 4]. These representations can be codified both by topological or topographic descriptors. Topological descriptors are the most popular because the 2D representation of molecules is computationally less difficult to work with than the $3 \mathrm{D}$ representation [1].

This work proposes a different approach in contrast with what is rigorously known as molecular similarity or chemical similarity [1]. The descriptor and the method of reduction of the graph used contain both structural and chemical-physical information. Thus, the approach allows evaluations and comparisons to be made by accounting 
for not only the structure but also other properties associated with the electrostatic nature of the molecule or fragment. The methods of structural similarity in 2D are more popular and simple. However, when working with only the topology of the molecules, most of the information associated with the spatial distribution is lost, except in the molecules that are essentially flat. As opposed to 2D methods, 3D methods consider that the properties of molecules tend to be strongly associated with the spatial distribution of their atoms $[5,6]$. On the other hand, the 3D methods based on 3D data usually compute a single conformation per molecule, which may not agree with the bioactive conformation. It is a common problem for all methods based on single conformation.

This issue causes a dilemma for researchers: losing all three-dimensional information for the sake of simplicity in the calculations or complicating the calculations and possibly delaying the results. The possibility of obtaining large data sets is an unquestionable reality. In that case, the eventual distortion of the $3 \mathrm{D}$ results due to not adjusting to the required conformation must be compensated by the increase in the number of compounds. However, such voluminous processing is not currently an impediment in terms of computational cost $[7,8]$.

Another concept that has been used for more than two decades is the scaffold and, more recently, scaffold hopping. These concepts allow the reduction of the molecule by eliminating R-substituents from the nucleus supposedly responsible for the activity in series of compounds in the first case, and in the other case, they allow the scaffold to be determined and enable comparisons to be made between structurally different compounds [9]. In other words, this approach bears a certain similarity to the proposed method since both seek to identify structurally different compounds that may show similar biological activity.

For these reasons, the proposed similarity method is based on the molecular description with a 3D descriptor that has structural information and on the polarity of the molecular graph or its fragments defined by a chemical graph reduction method.

Furthermore, molecular similarity based on substructure allows obtaining the molecular fragment or common subgraph among pairs of compounds [10, 11]. Several similarity methods have been developed based on a group of algorithms aimed at obtaining the largest common subgraph among a pair of compounds, the maximum common subgraph (MCS) [12-14]. To quantify the molecular similarity, this method uses the Tanimoto coefficient $\left(T c_{M C S}\right)[15,16]$.

In this work, we introduce a new concept called maximum common property (MCPhd), inspired by MCS, to quantify the similarity based on substructure, using the electrotopographic state index for atoms (Sstate $3 D$ ) [17], which was developed from its parent electrotopologic defined by Kier and Hall [18] from the connectivity matrix of the hydrogen-depleted chemical graph as an atomic descriptor.

The rest of the paper is organized in sections as follows: Related Works describes several relevant and recent proposals related to this work; Materials and Methods describes the dataset and molecular codification, the general procedure and the proposed MCPhd algorithm; Results and Discussion describes the experimental results; and finally, Conclusions presents a summary of this work.

\section{Related works}

In the SAR and QSAR approaches, the similarity between molecular structures is measured from some fragments of structural interest, physico-chemical properties, or other characteristics that are relevant to the biological activity under study. Therefore, the quality in the description and representation of molecular structures is a very important issue in the construction of computational models [19].

There are several proposals that consider the 3D information of the structure to calculate the similarity between chemical compounds. For example, Raymond and Willett [20] proposed a 3D MCSs method for similarity searching based on finding the largest set of atoms common to both molecules that preserves all pairwise distance constraints in both molecules. Although the number of freedom rotational degree is usually a difficulty, it was solved by generating several conformations. In order to establish the maximum and minimum possible distances between all pairs of atoms in a molecule, they applied the distance geometry described by Crippen et al. [21]. This procedure shows a computational complexity of $O\left(N^{3}\right)$.

Other 3D similarity methods like LS-align [22], generate atom-level structural alignments of ligand molecules, by an iterative heuristic search of the target function that combines inter-atom distance with mass and chemical bond comparisons.

Shape-feature similarity (SHAFTS) [23] is a hybrid approach for 3D molecular similarity calculation. The method adopts a hybrid similarity metric combined with molecular shape and colored (labeled) chemistry groups annotated by pharmacophore features for 3D similarity calculation. The method needs molecular alignments and superpositions between the target and the query molecules.

The ligand-based approach LigCSRre [24] uses 3D structural data of molecules for similarity studies. It combines a 3D maximum common substructure search algorithm independent from atom order with a tunable description of atomic compatibilities to prune the search.

3D similarity is attracting attention of the scientific community. Many methods to describe the shape of molecules have been developed. Surface-based approaches such as 3D Zernike descriptors and others demonstrated 
a good virtual screening performance [25]. Futhermore, nowadays there is a wide variety of web services, source code libraries and frameworks such as Open Babel [26], CoSiAn [27], ChemMapper [28], SMSD Toolkit [29], Corina [30], ISIDA-Platform [31], Chemaxon Web Services [32], and Chemical Development Kit (CDK) library [33] that allow to calculate 2D and 3D descriptors, build and validate QSAR models, and support the implementation of new computational models and algorithms.

\section{Materials and methods}

\section{Sample used}

We employed a set of 4-aminobicyclo[2.2.2]octan-2-yl 4-aminobutanoates (Table 1 ) reported by Weis et al. [34] and evaluated compounds against the multiresistant K-1 strain of Plasmodium falciparum.

\section{Codification of structures}

The electrotopographic state index for atoms [17] was used to codify chemical structures. This index is defined by Eq. (1).

$$
\text { Sstate }_{3 D}=I_{i}+\triangle I_{i j}
$$

where Sstate $_{3 D}$ is the calculated value of the atom $i$ in the corresponding molecule and $I_{i}$ is the intrinsic value of the atom $i$ calculated with Eq. (2).

$$
I_{i}=\left[(2 / N) 2_{v}+1\right] / \delta
$$

where $N$ is the principal quantum number of atom $I, \delta^{v}$ is the number of valence electrons in the molecular skeleton $\left(Z^{v}-\mathrm{h}\right)$ and $\delta$ is the number of $\sigma$ electrons in the skeleton $(\sigma-h)$. For each atom of the molecular skeleton, $\delta^{v}$ is the number of valence electrons, $\sigma$ is the number of electrons in $\sigma$ orbitals and $h$ is the number of hydrogen atoms bonded.

$\triangle I_{i j}$ represents the disturbance of the atoms of the environment, which is calculated by Eq. (3).

$$
\Delta I_{i j}=\sum\left(I_{i}+I_{j}\right) / r_{i j}^{2}
$$

where the sum is over the difference of the intrinsic values of atom i with respect to each one of the other atoms in the molecule and $r_{i j}^{2}$ is the Euclidean distance between the analyzed atoms, transforming the original topological index of Kier and Hall in topographic.

\section{Graph reduction}

The reduction of the chemical graph is carried out by the method described by Carrasco et al. [35], where the descriptor centers (DCs), rings of different orders ( $\mathrm{Rn})$, clusters of order 3 and 4 (C3 and C4, respectively), heteroatoms such as halogens, amino, etc. (X), and terminal groups such as methyl $\left(M_{3}\right)$, methylene $\left(M_{2}\right)$ and methyne (M) are defined. Examples of these parameters are shown in Fig. 1. This graph reduction procedure, named CALEDE, is inspired by the procedure developed by Avindon et al. [36], where each DC is assigned the total value of Sstate $_{3 D}$, quantified as the sum of the value of Sstate $3 D i$ of each atom that conforms to it.

\section{Definition of the maximum common property}

The maximum common property (MCPhd) between two fully connected and complete (not hydrogen-depleted) $G_{1}$ and $G_{2}$ chemical graphs is defined as the maximum similarity in the chemical-physical properties represented by the index Sstate $_{3 D}$, which exists between subgraphs $g_{1}$ and $g_{2}$ of the molecular graphs $G_{1}$ and $G_{2}$, respectively. Both $g_{1}$ and $g_{2}$ represent the link of at least two DCs that are at a Euclidean distance $\mathrm{dE}\left(D C_{1}, D C_{2}\right)$ from their corresponding centers of mass from pairs of DCs.

To quantify the value of similarity between two compounds using the concept of the maximum common property (MCPhd), the calculation of the similarity of two compounds is assumed using the Tanimoto function or coefficient on the basis of the maximum common substructure called $T c_{M C S}[15,16]$. The $T c_{M C S}$ for two molecules $\mathrm{A}$ and $\mathrm{B}$ is defined as:

$$
T c_{M C S}=\frac{|M C S(A, B)|_{b}}{|A|_{b}+|B|_{b}-|M C S(A, B)|_{b}}
$$

where $|A|_{b}$ is the number of links of $\mathrm{A},|B|_{b}$ is the number of links of $\mathrm{B}$ and $|M C S(A, B)|_{b}$ is the number of links of the MCS of A and B. If the concept MCPhd is replaced in Eq. (4), it yields:

$$
T c_{M C P h d}=\frac{|M C P h d(A, B)|_{b}}{|A|_{b}+|B|_{b}-|M C P h d(A, B)|_{b}}
$$

where $|A|_{b}$ is the number of heavy atoms of $\mathrm{A},|B|_{b}$ the number of heavy atoms of $\mathrm{B}$ and $|\operatorname{MCPhd}(A, B)|_{b}$ the smallest number of heavy atoms among the fragments with the highest MCPhd between A and B.

\section{The proposed MCPhd algorithm}

Figure 2 shows the algorithm used for the calculation of similarity. The algorithm uses the following parameters: $\left(G_{1}\right.$ and $\left.G_{2}\right)$ two compounds or molecules, (u) the similarity threshold, (f) the similarity coefficient and (i) the index used to quantify the similarity. First, we obtain the subgraphs $\left(f_{1}\right.$ and $\left.f_{2}\right)$ that have a maximum common property value quantified by the index based on the parameters and similarity coefficient. These subgraphs are obtained by performing the following steps:

1 The index (i) entered as a parameter is calculated for each atom in each $G_{1}$ and $G_{2}$ graph using the Chemi- 
Table 1 Compounds set

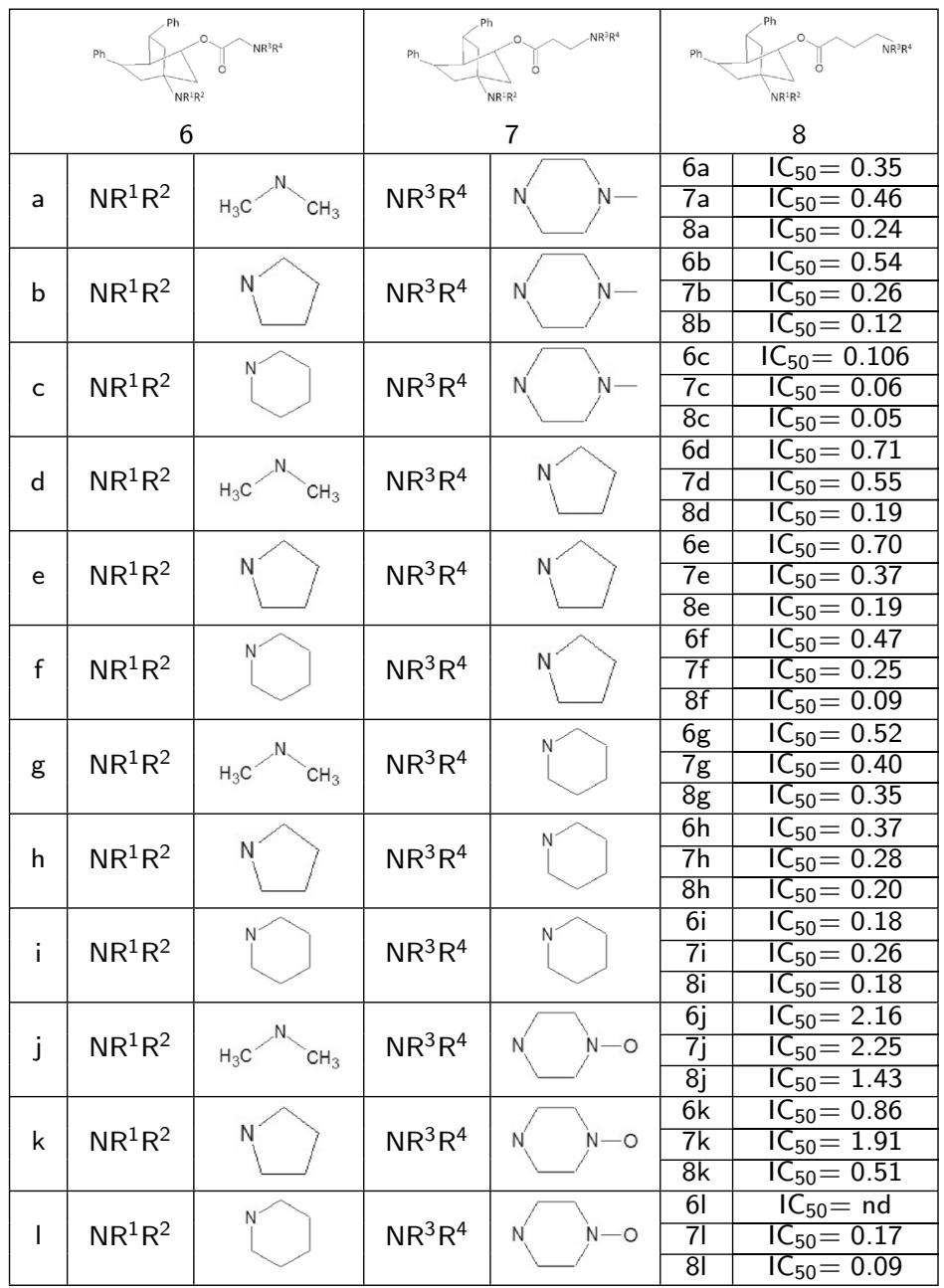

cal Development Kit (CDK) library [33]. Lines 1 and 2 of the algorithm are shown in Fig. 2.

2 The graphs $\left(G_{1}\right.$ and $\left.G_{2}\right)$ on DCs are reduced, and the total index value of each one is obtained. Lines 3 and 4 of the algorithm are shown in Fig. 2.

3 The similarity matrix between the DCs obtained from the graphs $\left(G_{1}\right.$ and $G_{2}$ ) is constructed using the similarity coefficient introduced as a parameter, along with the distance matrix between the DCs of each graph $\left(G_{1}\right.$ and $\left.G_{2}\right)$ using the Euclidean distance. Line 5 of the algorithm is shown in Fig. 2.

4. The DCs from each graph $\left(G_{1}\right.$ and $\left.G_{2}\right)$ that meet the condition that the similarity value must be higher than the similarity threshold $(u)$, entered as a parameter, are selected. Line 5 of the algorithm is shown in Fig. 2.

5 Finally, using the list of DCs obtained in the previous step and the distance matrices of the DCs in graph
$G_{1}$ and $G_{2}$, a new distance matrix between pairs of DCs in each graph $G_{1}$ and $G_{2}$ is constructed using the Canberra distance coefficient [38], as shown in Fig. 3. Then for each pair of DCs selected, a list is created in which the pairs of DCs in the created matrix whose distance is less than or equal to 0.15 are stored. Finally, the largest lists is selected and from each one the subgraphs $f_{1}$ and $f_{2}$ are generated. Line 5 of the algorithm is shown in Fig. 2

Then, for a pair of subgraphs $\left(f_{1}\right.$ and $\left.f_{2}\right)$ obtained and the graphs $\left(G_{1}\right.$ and $\left.G_{2}\right)$, the values of the variables needed to quantify similarity are obtained using the similarity coefficient $(\mathrm{u})$ for the discrete data entered as a parameter. Variable $\mathrm{c}$ is assigned the least number of heavy atoms belonging to the subgraphs ( $f_{1}$ and $f_{2}$ ), while variables a and $\mathrm{b}$ are assigned the number of heavy atoms belonging to each graph $\left(G_{1}\right.$ and $\left.G_{2}\right)$, respectively. Finally, these 


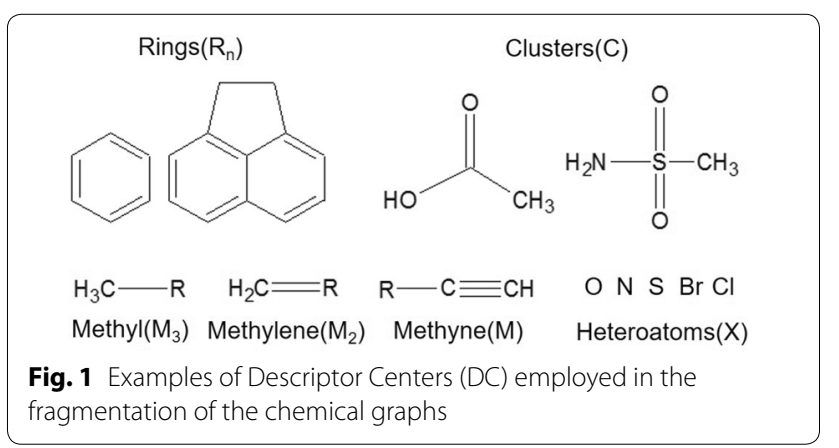

values are substituted in the similarity function to obtain the quantification of the similarity of the graphs $\left(G_{1}\right.$ and $G_{2}$ ). Lines 6 to 16 of the algorithm are shown in Fig. 2. Furthermore, if there are several subgraphs $f_{1}$ and $f_{2}$, the same operation is performed for each one and the pair of subgraphs $f_{1}$ and $f_{2}$ with the highest similarity value is selected.

The use of the algorithm is exemplified below using the molecules $6 \mathrm{k}$ and $6 \mathrm{c}$ present in the dataset as shown in Figs. 4 , 5, respectively. We use 5 parameters $\left(G_{1}, G_{2}, u, f, i\right)$ for its operation, where $G_{1}$ and $G_{2}$ are the molecular graphs $6 \mathrm{k}$ and $6 \mathrm{c}$ respectively, $(i)$ is the index $\left(\right.$ Sstate $\left._{3 D}\right),(u)$ is the similarity threshold, and $(f)$ is the similarity function. For this example, we will use 0.95 and the modified Tanimoto coefficient $\left(T c_{M C P h d}\right)$ as the threshold and similarity function, respectively. Then, after assigning the parameters, the following steps are performed:

1 The Sstate $_{3 D}$ index is calculated for each atom present in molecules $6 \mathrm{k}$ and $6 \mathrm{c}$; these results are shown in Tables 2 and 3.

2 The $6 \mathrm{k}$ and $6 \mathrm{c}$ molecular graphs on DCs are reduced, and each is given the value of the total Sstate $_{3 D}$ index. As shown in step A of Fig. 6, molecule 6k is reduced on the DCs $\left(R 8_{1}, R 5_{2}, R 6_{3}, R 6_{4}, R 6_{5}, C 3_{6}, X_{7}\right)$, while molecule $6 \mathrm{c}$ is reduced on $\left(R 6_{1}, R 8_{2}, R 6_{3}, R 6_{4}, R 6_{5}\right.$, $\left.C 3_{6}, M_{7}\right)$.

3 The similarity matrix between the DCs of each molecule $6 \mathrm{k}$ and $6 \mathrm{c}$ is constructed using the continuous Tanimoto coefficient (Tc) [37], together with the distance matrices between the DCs of each molecule (6k and 6c), as shown in step B of Fig. 6.

4. DCs are selected from each molecule (6k and 6c) that meet the condition that the similarity value is above the similarity threshold of 0.95 . The DCs selected

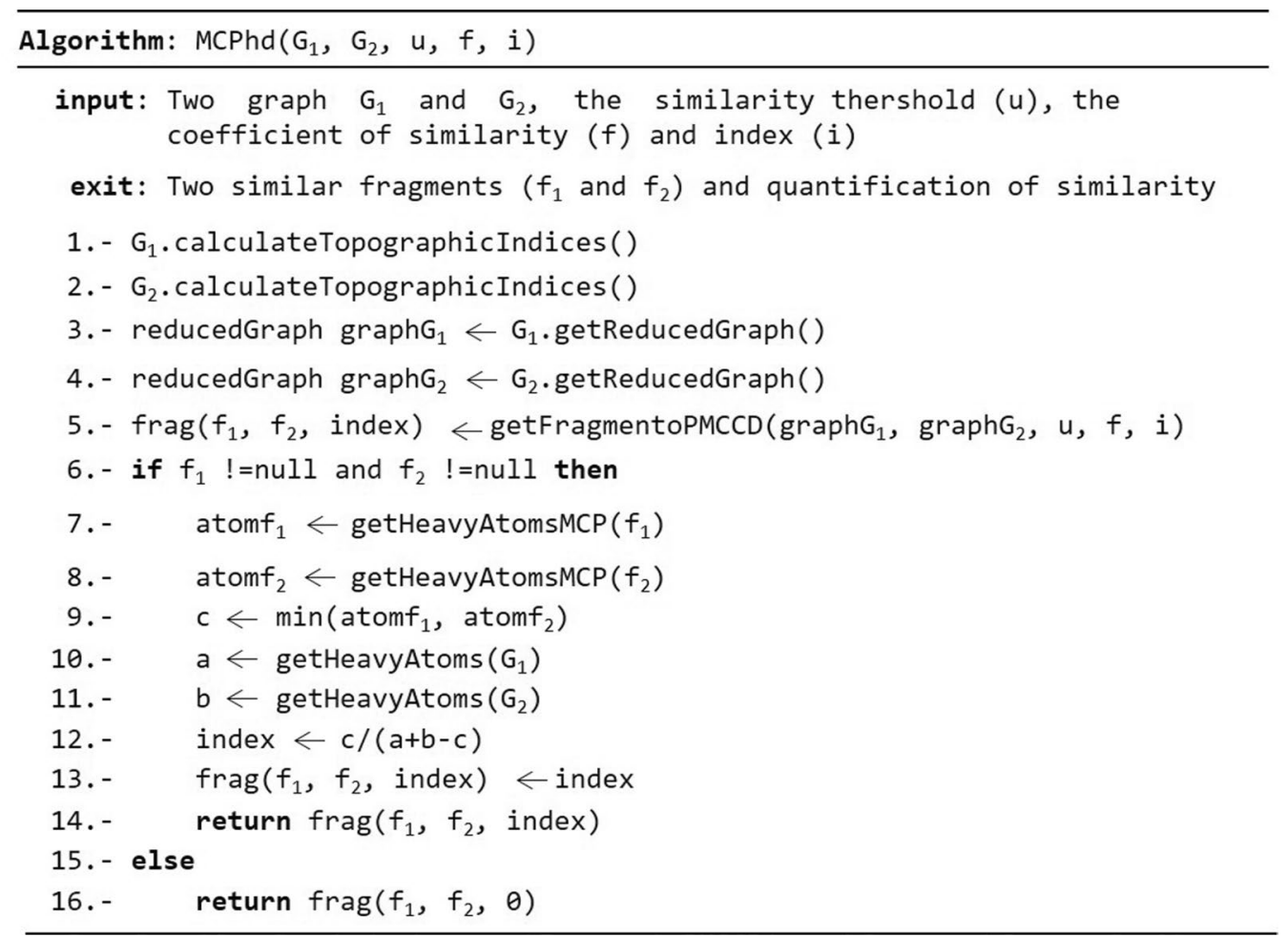

Fig. 2 MCPhd algorithm for similarity calculation 


$$
d_{A}=\left(\begin{array}{lll}
d_{D C_{A 1}, D C_{A 1}} & d_{D C_{A 1}, D C_{A 2}} & d_{D C_{A 1}, D C_{A 3}} \\
d_{D C_{A 2}, D C_{A 1}} & d_{D C_{A 2}, D C_{A 2}} & d_{D C_{A 2}, D C_{A 3}} \\
d_{D C_{A 3}, D C_{A 1}} & d_{D C_{A 3}, D C_{A 2}} & d_{D C_{A 3}, D C_{A 3}}
\end{array}\right) \quad d_{B}=\left(\begin{array}{lll}
d_{D C_{B 1}, D C_{B 1}} & d_{D C_{B 1}, D C_{B 2}} & d_{D C_{B 1}, D C_{B 3}} \\
d_{D C_{B 2}, D C_{B 1}} & d_{D C_{B 2}, D C_{B 2}} & d_{D C_{B 2}, D C_{B 3}} \\
d_{D C_{B 3}, D C_{B 1}} & d_{D C_{B 3}, D C_{B 2}} & d_{D C_{B 3}, D C_{B 3}}
\end{array}\right)
$$

Pairs of similar DCs: $\mathrm{DC}_{\mathrm{A} 1}-\mathrm{DC}_{\mathrm{B} 1}, \mathrm{DC}_{\mathrm{A} 2}-\mathrm{DC}_{\mathrm{B} 3}, \mathrm{DC}_{\mathrm{A} 3}-\mathrm{DC}_{\mathrm{B} 2}$

c) Distance matrix between pairs DCs

$$
\begin{gathered}
d_{(A, B)}=\left(\begin{array}{lll}
d_{d_{D C_{A 1}, D C_{A 1}} ; d_{D C_{B 1}, D C_{B 1}}} & d_{d_{D C_{A 1}, D C_{A 2}} ; d_{D C_{B 1}, D C_{B 3}}} & d_{d_{D C_{A 1}, D C_{A 2}} ; d_{D C_{B 1}, D C_{B 2}}} \\
d_{d_{D C_{A 2}, D C_{A 1}} ; d_{D C_{B 3}, D C_{B 1}}} & d_{d_{D C_{A 2}, D C_{A 2}} ; d_{D C_{A 3}, D C_{A 3}}} & d_{d_{D C_{A 2}, D C_{A 3}} ; d_{D C_{B 3}, D C_{B 2}}} \\
d_{d_{D C_{A 3}, D C_{A 1} ;} ; d_{D C_{B 2}, D C_{B 1}}} & d_{d_{D C_{A 3}, D C_{A 2}} ; d_{D C_{B 2}, D C_{B 3}}} & d_{d_{D C_{A 3}, D C_{A 3}} ; d_{D C_{B 2}, D C_{B 2}}}
\end{array}\right) \\
d_{d_{D C_{A 3}, D C_{A 1} ;} ; d_{D C_{B 2}, D C_{B 1}}}=\frac{\left|d_{D C_{A 3}, D C_{A 1}}-d_{D C_{B 2}, D C_{B 1} \mid}\right|}{\left|d_{D C_{A 3}, D C_{A 1}}\right|+\left|d_{D C_{B 2}, D C_{B 1}}\right|}
\end{gathered}
$$

Fig. 3 Distance matrix between pairs of similar DCs

Table 2 Result of the Sstate $_{3 D}$ calculation for each atom of the molecule $6 \mathbf{k}$

\begin{tabular}{lccllc}
\hline \multicolumn{7}{l}{ Molecule 6k } & & & & \\
\hline Atom & Number & Sstate3D & Atom & Number & Sstate3D \\
\hline C & 1 & 0.27653 & N & 19 & -1.4592 \\
C & 2 & 4.24474 & $\mathrm{C}$ & 20 & 3.38006 \\
$\mathrm{C}$ & 3 & -2.11427 & $\mathrm{C}$ & 21 & 3.11542 \\
$\mathrm{C}$ & 4 & 4.25231 & $\mathrm{C}$ & 22 & -0.12941 \\
$\mathrm{C}$ & 5 & 0.49396 & $\mathrm{C}$ & 23 & 3.09432 \\
$\mathrm{C}$ & 6 & 0.52371 & $\mathrm{C}$ & 24 & 3.32881 \\
$\mathrm{C}$ & 7 & 0.28273 & $\mathrm{O}$ & 25 & 4.22234 \\
$\mathrm{C}$ & 8 & 4.2063 & $\mathrm{~N}$ & 26 & -1.30847 \\
$\mathrm{C}$ & 9 & -0.37931 & $\mathrm{C}$ & 27 & 3.52697 \\
$\mathrm{C}$ & 10 & 3.51969 & $\mathrm{C}$ & 28 & 2.9831 \\
$\mathrm{C}$ & 11 & 2.98731 & $\mathrm{C}$ & 29 & 3.01061 \\
$\mathrm{C}$ & 12 & 2.89649 & $\mathrm{C}$ & 30 & 3.54754 \\
$\mathrm{C}$ & 13 & 2.99502 & $\mathrm{C}$ & 31 & -0.38433 \\
$\mathrm{C}$ & 14 & 3.5348 & $\mathrm{C}$ & 32 & 3.54001 \\
$\mathrm{O}$ & 15 & -0.08644 & $\mathrm{C}$ & 33 & 2.99825 \\
$\mathrm{C}$ & 16 & -0.10545 & $\mathrm{C}$ & 34 & 2.90069 \\
$\mathrm{O}$ & 17 & 5.5644 & $\mathrm{C}$ & 35 & 2.99544 \\
$\mathrm{C}$ & 18 & 3.75612 & $\mathrm{C}$ & 36 & 3.53921 \\
\hline
\end{tabular}

from molecules $6 \mathrm{k}$ and $6 \mathrm{c}$ are $\left(R 8_{1}, R 5_{2}, R 6_{3}, R 6_{4}\right.$, $R 6_{5}$ and $\left.C 3_{6}\right)$ and $\left(R 6_{1}, R 8_{2}, R 6_{3}, R 6_{4}, R 6_{5}, C 3_{6}\right.$ and $\left.M 3_{7}\right)$, respectively, as shown in step $\mathrm{C}$-a in Fig. 6. Furthermore, using the distance matrices of the graphs obtained in the previous step, for each pair of DCs, a list is constructed with the pairs of DCs that
Table 3 Result of the Sstate $_{3 D}$ calculation for each atom of the molecule $6 c$

\begin{tabular}{lccllc}
\hline \multicolumn{2}{l}{ Molecule 6c } & & & & \\
\hline Atom & Number & Sstate3D & Atom & Number & Sstate3D \\
\hline C & 1 & 0.35527 & $\mathrm{C}$ & 20 & 3.314 \\
$\mathrm{C}$ & 2 & 4.17197 & $\mathrm{C}$ & 21 & 2.96595 \\
$\mathrm{C}$ & 3 & -2.19195 & $\mathrm{~N}$ & 22 & -2.22668 \\
$\mathrm{C}$ & 4 & 4.25527 & $\mathrm{C}$ & 23 & 2.94306 \\
$\mathrm{C}$ & 5 & 0.37013 & $\mathrm{C}$ & 24 & 3.25612 \\
$\mathrm{C}$ & 6 & 0.50352 & $\mathrm{C}$ & 25 & 8.9881 \\
$\mathrm{C}$ & 7 & 0.21375 & $\mathrm{~N}$ & 26 & -1.46525 \\
$\mathrm{C}$ & 8 & 4.23211 & $\mathrm{C}$ & 27 & 3.52712 \\
$\mathrm{C}$ & 9 & -0.24053 & $\mathrm{C}$ & 28 & 2.94719 \\
$\mathrm{C}$ & 10 & 3.50889 & $\mathrm{C}$ & 29 & 2.85191 \\
$\mathrm{C}$ & 11 & 3.00512 & $\mathrm{C}$ & 30 & 2.95634 \\
$\mathrm{C}$ & 12 & 2.94959 & $\mathrm{C}$ & 31 & 3.54763 \\
$\mathrm{C}$ & 13 & 3.09722 & $\mathrm{C}$ & 32 & -0.13708 \\
$\mathrm{C}$ & 14 & 3.69435 & $\mathrm{C}$ & 33 & 3.3945 \\
O & 15 & -0.48635 & $\mathrm{C}$ & 34 & 2.9161 \\
$\mathrm{C}$ & 16 & -0.31876 & $\mathrm{C}$ & 35 & 2.85145 \\
$\mathrm{O}$ & 17 & 5.6053 & $\mathrm{C}$ & 36 & 2.96557 \\
$\mathrm{C}$ & 18 & 3.73854 & $\mathrm{C}$ & 37 & 3.52272 \\
$\mathrm{~N}$ & 19 & -1.66555 & & & \\
\hline
\end{tabular}

are at a Canberra distance less than or equal to 0.15 , as shown in step C-b in Fig. 6.

5 From the lists of DC pairs obtained in the previous step, the following DCs are selected, namely, $\left(R 8_{1}\right.$, $R 5_{2}, R 6_{4}$ and $\left.C 3_{6}\right)$ and $\left(R 6_{1}, R 8_{2}, R 6_{3}\right.$ and $\left.C 3_{4}\right)$, cor- 


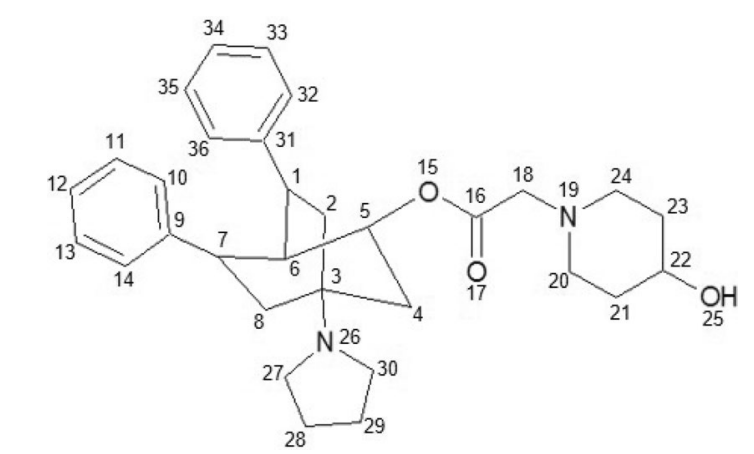

Fig. $42 \mathrm{D}$ graph of the $6 \mathrm{k}$ molecule

responding to the lists $(1,3,4$ and 5$)$ according to the larger size list with the same DCs in common.

Finally, the similarity value of the two molecules $6 \mathrm{k}$ and $6 \mathrm{c}$ is quantified using the modified Tanimoto coefficient $\left(T c_{M C P h d}\right)$, where the value of $|M C P h d(A, B)|_{b}$ is the lowest number of heavy bonds present between fragments $f_{1}$ and $f_{2}$, while the values of $|A|_{b}$ and $|B|_{b}$ are obtained from the number of heavy atoms present in molecules $6 \mathrm{k}$ and $6 \mathrm{c}$, respectively. With these values, it is possible to quantify the similarity between molecules $6 \mathrm{k}$ and $6 \mathrm{c}$. In step $\mathrm{E}$ of Fig. 6, it can be seen that the number of heavy atoms of fragments $f_{1}$ and $f_{2}$ is 23 and 24, respectively, so the value of $|\operatorname{MCPhd}(A, B)|_{b}$ is 23 , while the number of heavy atoms of molecules $6 \mathrm{k}$ and $6 \mathrm{c}$ is 36 and 37, respectively; that is, $|A|_{b}=36$ and $|B|_{b}=37$. Therefore, the calculated value of similarity between molecules $6 \mathrm{k}$ and $6 \mathrm{c}$ is 0.46 .

\section{Small molecule subgraph MCS approach}

The Small Molecule Subgraph Detector (SMSD) algorithm differs from previous MCS algorithms in that it uses a combination of several algorithms to find the common maximum subset and filters the results in a way that is chemically relevant because it incorporates chemical knowledge (coincidence of atom type with information sensitive and insensitive to the bond) while searching for molecular similarity. In addition, the algorithm calculates the maximum subgraph common between two molecules (A and B) by combining the power of the VFLibMCS, MCSPlus and CDKMCS algorithms. These algorithms are used on a case-by-case basis, depending on the molecules under consideration for the common maximum subgraph search [29]. This algorithm is implemented in the SMSD tool available

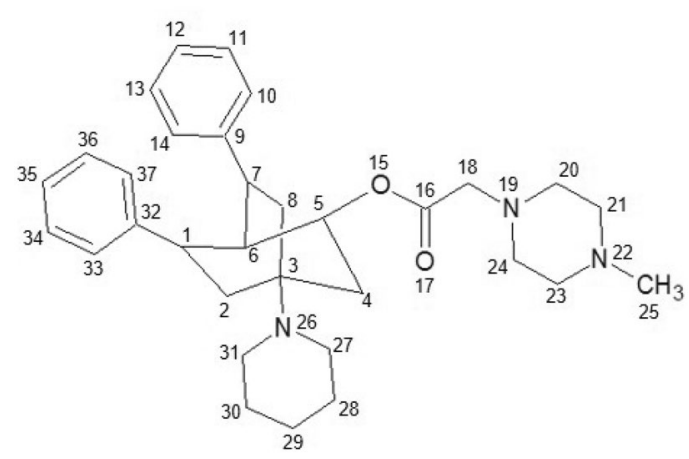

Fig. 5 2D graph of the $6 \mathrm{c}$ molecule

free of charge on the official site of the European Institute of Bioinformatics.

\section{General experimental procedure}

The experiments were carried out as shown in Fig. 7, based on a test of 36 compounds with a 2D structure, which have been tested experimentally in the study conducted by Weis et al. in 2014 [34]. The 3D structure of each compound was obtained through the Corina online service [30]. The $2 \mathrm{D}$ structures were used to calculate the molecular similarity (all against all) with the SMSD, OBabel_FP2 and ISIDA algorithms, while the $3 \mathrm{D}$ structures were processed to calculate the Sstate $_{3 D}$ index of each atom and to reduce their graphs on DCs in order to apply the MCPhd algorithm to calculate the molecular similarity (all against all), and to use the SHAFTS method. The similarity was calculated using the OBabel_FP2, SHAFTS and ISIDA methods through the web service CoSiAn (Combinatorial Similarity Analysis) [27] and ChemMapper [28]. Additional file 1 contains all necessary data/files to reproduce the results.

To quantify the value of similarity between different $I C_{50}$ we defined the following coefficient $T_{c} I C_{50}$ based on continuous Tanimoto [37]:

$$
\operatorname{TcIC}_{50}=\frac{A_{I C_{50}} * B_{I C_{50}}}{\left(A_{I C_{50}}\right)^{2}+\left(B_{I C_{50}}\right)^{2}-\left(A_{I C_{50}} * B_{I C_{50}}\right)}
$$

where $A_{I C_{50}}$ and $B_{I C_{50}}$ are the $I C_{50}$ value of $\mathrm{A}$ and $\mathrm{B}$ respectively.

Finally, the results obtained by all the algorithms were compared from different perspectives: (i) the statistical difference of the MCPhd results with respect to those obtained by the other methods; (ii) the ratio of the similarity values obtained by the different methods against the values obtained for $T c I C_{50}$; (iii) the percentage of 


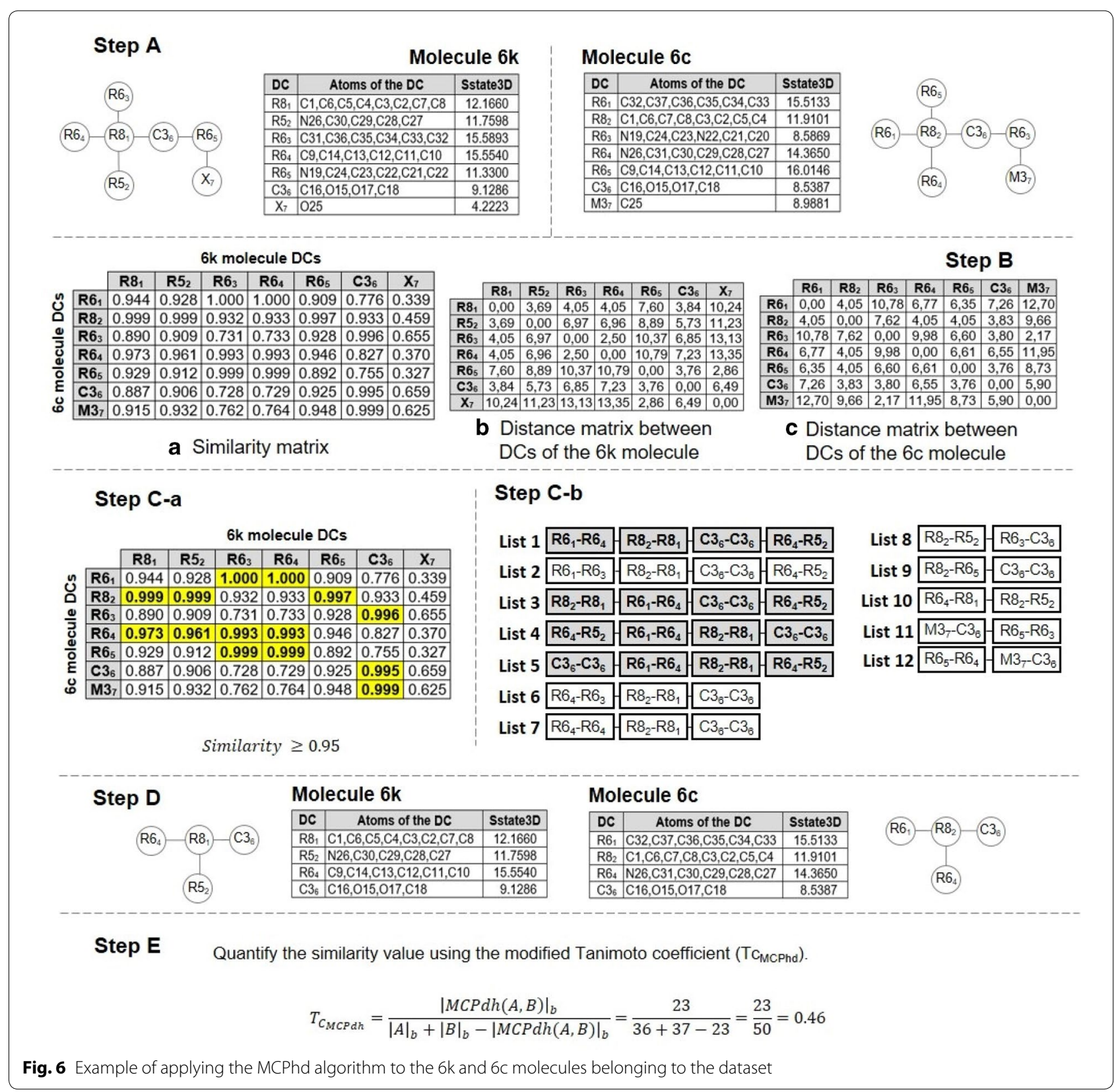

success for the different similarity methods to find structures with the same activity, similar to a screening process; (iv) the results of the different methods in relation to the concept of bioisosterism or the analogy between the physicochemical properties of the molecular fragments; (v) the computational cost.

The MCPhd algorithm was implemented using the JAVA language and CDK library, all test were executed on an Intel(R) Core(TM) i7-7500U PC with 16 GB of RAM.

\section{Results and discussion}

The molecular similarity methods compared in this work, SMSD OBabel_FP2, ISIDA, SHAFTS and MCPhd, use different approaches to quantify the similarity between two molecular graphs or molecules. Whereas SMSD employs graph isomorphism and no other properties associated to the molecular structure, OBabel_FP2 uses the similarity between hashed fingerprints that represent molecule substructures, ISIDA employs substructural 
molecular fragments, and SHAFTS adopts a hybrid similarity metric combined with molecular shape and colored chemistry groups for 3D molecular similarity calculation. The similarity calculated with MCPhd is based on the criterion of analogy or proximity between the physicochemical properties of the molecular fragments or subgraphs that are compared, expressing these properties as an Sstate $_{3 D}$ value.

As we will show, this approach places MCPhd closer to the concepts of bioisosterism. Bioisosterism denote that two different molecules can afford similar biological responses if the structural features are accomplished by physicochemical property that is responsible in great measure of the biological response. This concept was coined by Friedman [39], extended by Burger [40] and recently used by Lassalas et al. [41] and Tahirova [42].

Using these different approaches, different similarity values were obtained. Table 4 shows the results of the comparison with the remaining 35 molecules of the sample, with compounds $8 \mathrm{c}$ and $7 \mathrm{j}$ used as target elements since they had the minimum and maximum $I C_{50}$ values, respectively.

To determine whether the results produced by the MCPhd methods are significantly different, a non-parametric statistical test is used for two independent MannWhitney samples [43] with a significance level of $5 \%$. The results of the Mann-Whitney $\mathrm{U}$ statistic and $p$ values (bilateral asymptotic significance) for the most active (8c) and least active (7j) compounds are shown in Table 5. As the $p$ values for both compounds are below 0.05 , it is concluded that the similarity values obtained by the MCPhd methods were significantly different from the other similarity methods.

Furthermore, it can be seen in Figs. 8, 9 for the most active compound (8c) and the least active compound (7j), respectively, that the results obtained by the similarity methods used showed a low correlation with the results achieved when applying the MCPhd method. Table 6 shows these results for all the active compounds in the dataset.

As the maximum inhibitory concentration $\left(I C_{50}\right)$ is a measure of a compound's efficacy in inhibiting biological or biochemical function, it is expected that compounds with near values of $I C_{50}$, are very similar and with far values of $I C_{50}$, the compounds will exhibit very low similarity. Under that hypothesis, the results were analyzed from another perspective. The similarity was calculated for the values of the variable $I C_{50}$ of the most active compound (8c) and the less active compound (7j) against the rest of the dataset. The results are shown in the $T c I C_{50}$ columns of Table 4.

Subsequently, the molecular similarities calculated by the different methods were compared with this new variable. As shown in Fig. 10, the similarity results obtained by the MCPhd method for the most active compound (8c) had a slope closer to that obtained with

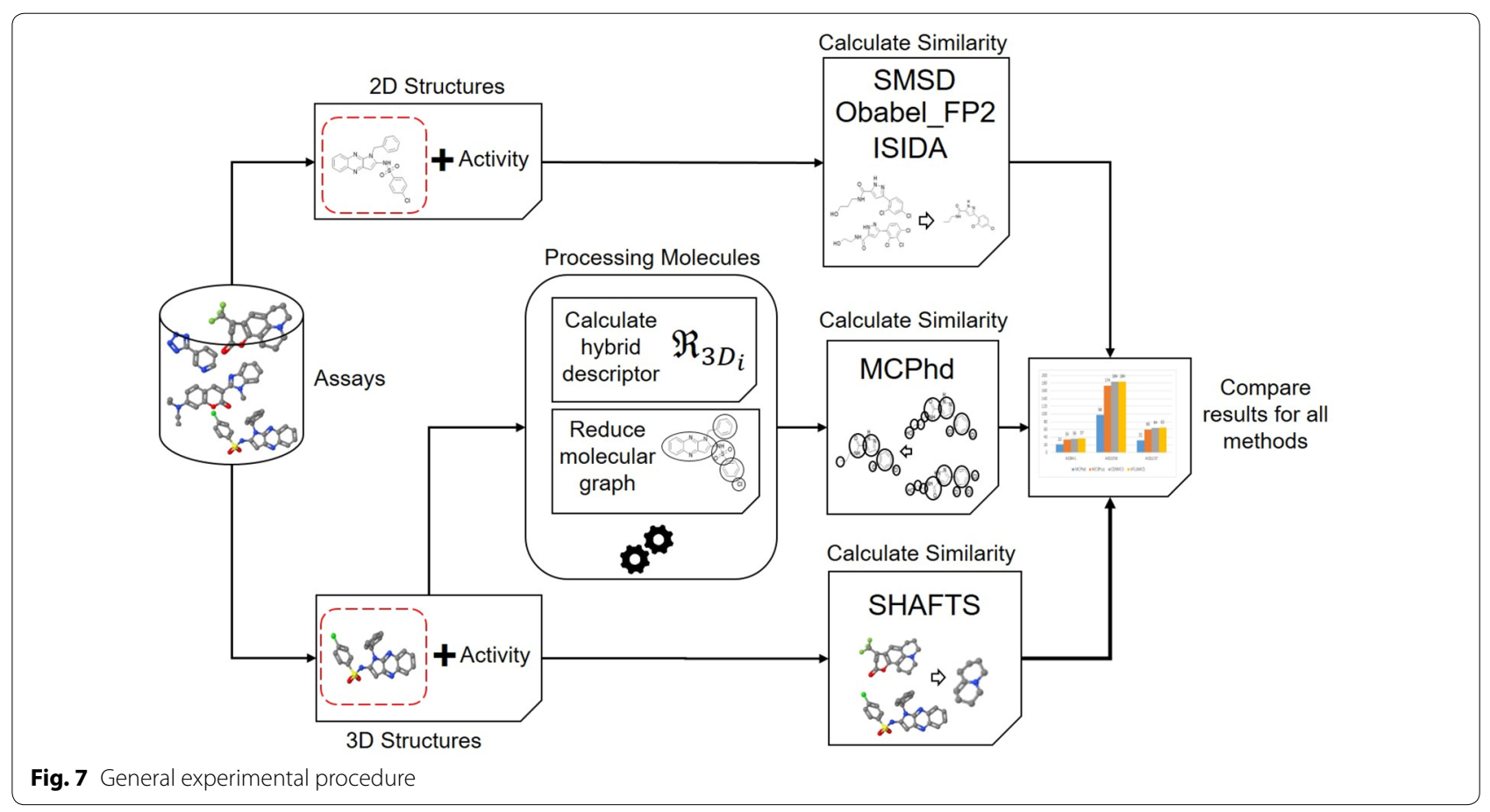


the $T c I C_{50}$ similarity; furthermore, the results were better correlated obtained the MCPhd method the best Pearson correlation coefficient $\left(r_{x y}=0.85\right)$ compared to the remaining methods as Fig. 11 shows.

A similar behavior was observed in the results obtained for the less active compound (7j). The slope of MCPhd was closer to TcIC 50 compared to the other methods (Fig. 12). The $r_{x y}=0.43$ of MCPhd vs. TcIC 50 was higher than the other similarity methods, with the exception of the SHAFTS method where $r_{x y}=0.55$ (see Fig. 13).
To generalize these results, the similarity obtained with all methods of the rest of the 17 compounds selected as active by Baptista [44] was correlated against $T c I C_{50}$. The results showed (Table 7) that overall the MCPhd method improved the correlation coefficient in $6 \%$ of the cases with respect to SHAFTS and $18 \%$ of the cases with respect to the remaining methods.

To perform a more exhaustive study comparing the molecular similarity results obtained by all methods, the following steps were performed: (1) the similarity is calculated with all methods for all compounds (one against

Table 4 Molecular similarity values of the most active and inactive compounds with the rest of the dataset

\begin{tabular}{|c|c|c|c|c|c|c|c|c|c|c|c|c|c|}
\hline \multicolumn{6}{|l|}{ Molecule 8c } & \multirow[t]{2}{*}{ Target } & \multirow[t]{2}{*}{$I C_{50}$} & \multicolumn{6}{|l|}{ Molecule 7j } \\
\hline OBabel_FP2 & SHAFTS & ISIDA & SMSD & MSChd & $\mathrm{TClC}_{50}$ & & & OBabel_FP2 & SHAFTS & ISIDA & SMSD & MSChd & $\mathrm{TclC}_{50}$ \\
\hline 1.00 & 1.00 & 1.00 & 1.00 & 1.00 & 1.00 & $8 c$ & 0.05 & 0.82 & 0.78 & 0.88 & 0.61 & 0.42 & 0.02 \\
\hline 0.90 & 1.00 & 0.97 & 0.67 & 0.97 & 0.97 & $7 c$ & 0.06 & 0.92 & 0.82 & 0.90 & 0.83 & 0.43 & 0.03 \\
\hline 0.91 & 1.00 & 0.97 & 0.95 & 0.77 & 0.74 & $8 f$ & 0.09 & 0.88 & 0.80 & 0.90 & 0.64 & 0.60 & 0.04 \\
\hline 0.92 & 1.00 & 0.90 & 0.90 & 0.70 & 0.74 & 81 & 0.09 & 0.89 & 0.77 & 0.97 & 0.61 & 0.35 & 0.04 \\
\hline 0.77 & 0.87 & 0.90 & 0.65 & 0.77 & 0.68 & $6 c$ & 0.106 & 0.74 & 0.73 & 0.81 & 0.60 & 0.29 & 0.05 \\
\hline 0.98 & 0.79 & 1.00 & 0.97 & 0.75 & 0.55 & $8 b$ & 0.12 & 0.80 & 0.88 & 0.88 & 0.62 & 0.33 & 0.06 \\
\hline 0.82 & 0.84 & 0.88 & 0.67 & 0.71 & 0.37 & 71 & 0.17 & 1.00 & 0.83 & 1.00 & 0.92 & 0.62 & 0.08 \\
\hline 0.72 & 0.87 & 0.87 & 0.67 & 0.53 & 0.35 & $6 i$ & 0.18 & 0.79 & 0.73 & 0.83 & 0.61 & 0.29 & 0.09 \\
\hline 0.92 & 1.00 & 0.97 & 0.93 & 0.75 & 0.35 & $8 i$ & 0.18 & 0.89 & 0.78 & 0.90 & 0.62 & 0.43 & 0.09 \\
\hline 0.89 & 0.76 & 0.97 & 0.87 & 0.46 & 0.33 & $8 d$ & 0.19 & 0.86 & 0.91 & 0.90 & 0.68 & 0.64 & 0.09 \\
\hline 0.90 & 0.93 & 0.97 & 0.92 & 0.56 & 0.33 & $8 \mathrm{e}$ & 0.19 & 0.87 & 0.77 & 0.90 & 0.65 & 0.42 & 0.09 \\
\hline 0.91 & 0.90 & 0.97 & 0.90 & 0.55 & 0.31 & $8 \mathrm{~h}$ & 0.20 & 0.88 & 0.74 & 0.90 & 0.64 & 0.33 & 0.09 \\
\hline 0.97 & 0.77 & 1.00 & 0.92 & 0.50 & 0.25 & $8 a$ & 0.24 & 0.79 & 0.87 & 0.88 & 0.65 & 0.69 & 0.12 \\
\hline 0.82 & 0.89 & 0.95 & 0.70 & 0.74 & 0.24 & $7 f$ & 0.25 & 0.99 & 0.77 & 0.92 & 0.87 & 0.51 & 0.12 \\
\hline 0.88 & 0.82 & 0.97 & 0.65 & 0.69 & 0.23 & $7 b$ & 0.26 & 0.90 & 0.76 & 0.90 & 0.85 & 0.33 & 0.13 \\
\hline 0.82 & 1.00 & 0.95 & 0.69 & 0.73 & 0.23 & $7 i$ & 0.26 & 1.00 & 0.82 & 0.92 & 0.89 & 0.44 & 0.13 \\
\hline 0.82 & 1.00 & 0.95 & 0.67 & 0.53 & 0.21 & $7 \mathrm{~h}$ & 0.28 & 0.99 & 0.83 & 0.92 & 0.92 & 0.34 & 0.14 \\
\hline 0.73 & 0.71 & 0.90 & 0.59 & 0.46 & 0.16 & $6 a$ & 0.35 & 0.69 & 0.87 & 0.81 & 0.64 & 0.64 & 0.18 \\
\hline 0.91 & 0.74 & 0.97 & 0.85 & 0.45 & 0.16 & $8 g$ & 0.35 & 0.88 & 0.88 & 0.90 & 0.67 & 0.71 & 0.18 \\
\hline 0.71 & 0.82 & 0.87 & 0.61 & 0.37 & 0.15 & $6 \mathrm{~h}$ & 0.37 & 0.78 & 0.75 & 0.83 & 0.63 & 0.43 & 0.19 \\
\hline 0.80 & 0.73 & 0.95 & 0.68 & 0.54 & 0.15 & $7 e$ & 0.37 & 0.97 & 0.94 & 0.92 & 0.89 & 0.35 & 0.19 \\
\hline 0.82 & 0.74 & 0.95 & 0.62 & 0.43 & 0.14 & $7 g$ & 0.40 & 1.00 & 0.84 & 0.92 & 0.97 & 0.73 & 0.21 \\
\hline 0.87 & 0.71 & 0.97 & 0.61 & 0.61 & 0.12 & $7 a$ & 0.46 & 0.88 & 0.91 & 0.90 & 0.89 & 0.71 & 0.24 \\
\hline 0.71 & 0.90 & 0.87 & 0.68 & 0.54 & 0.12 & $6 f$ & 0.47 & 0.78 & 0.75 & 0.83 & 0.63 & 0.46 & 0.25 \\
\hline 0.91 & 0.91 & 0.90 & 0.88 & 0.54 & 0.11 & $8 k$ & 0.51 & 0.88 & 0.72 & 0.97 & 0.62 & 0.35 & 0.27 \\
\hline 0.70 & 0.71 & 0.87 & 0.60 & 0.38 & 0.11 & $6 \mathrm{~g}$ & 0.52 & 0.77 & 0.88 & 0.83 & 0.66 & 0.66 & 0.28 \\
\hline 0.76 & 0.76 & 0.90 & 0.63 & 0.56 & 0.10 & $6 b$ & 0.54 & 0.73 & 0.83 & 0.81 & 0.61 & 0.31 & 0.29 \\
\hline 0.80 & 0.75 & 0.95 & 0.64 & 0.44 & 0.10 & $7 d$ & 0.55 & 0.97 & 0.87 & 0.92 & 0.94 & 0.74 & 0.30 \\
\hline 0.70 & 0.86 & 0.87 & 0.62 & 0.38 & 0.08 & $6 e$ & 0.70 & 0.77 & 0.77 & 0.83 & 0.64 & 0.33 & 0.40 \\
\hline 0.68 & 0.74 & 0.87 & 0.61 & 0.39 & 0.08 & $6 d$ & 0.71 & 0.75 & 0.91 & 0.83 & 0.68 & 0.68 & 0.40 \\
\hline 0.71 & 0.70 & 0.81 & 0.63 & 0.36 & 0.06 & $6 k$ & 0.86 & 0.78 & 0.85 & 0.90 & 0.61 & 0.37 & 0.50 \\
\hline 0.92 & 0.75 & 0.90 & 0.83 & 0.42 & 0.04 & 8j & 1.43 & 0.89 & 0.85 & 0.97 & 0.65 & 0.65 & 0.83 \\
\hline 0.82 & 0.70 & 0.88 & 0.65 & 0.52 & 0.03 & $7 \mathrm{k}$ & 1.91 & 0.99 & 0.99 & 1.00 & 0.95 & 0.50 & 0.97 \\
\hline 0.71 & 0.70 & 0.81 & 0.59 & 0.38 & 0.02 & $6 j$ & 2.16 & 0.78 & 0.86 & 0.90 & 0.64 & 0.64 & 1.00 \\
\hline 0.82 & 0.78 & 0.88 & 0.61 & 0.42 & 0.02 & $7 \mathrm{j}$ & 2.25 & 1.00 & 1.00 & 1.00 & 1.00 & 1.00 & 1.00 \\
\hline
\end{tabular}


Table 5 Mann-Whitney test values between the MCPhd method with the remainder on the most active and inactive compounds in the dataset

\begin{tabular}{lcllll}
\hline Methods & \multicolumn{2}{l}{ Molecule 8c } & & \multicolumn{2}{l}{ Molecule 7j } \\
\cline { 2 - 3 } \cline { 5 - 5 } & $\begin{array}{l}\text { U of Mann- } \\
\text { Whitney }\end{array}$ & $\begin{array}{l}\mathbf{p} \text { (Sig. Asint. } \\
\text { Bilateral) }\end{array}$ & $\begin{array}{l}\text { U of Mann- } \\
\text { Whitney }\end{array}$ & $\begin{array}{l}\mathbf{p} \text { (Sig. } \\
\text { Asint. } \\
\text { Bilateral) }\end{array}$ \\
\hline MCPhd & & & & \\
OBabel_FP & 131.50 & 0.00 & 33.00 & 0.00 \\
SHAFTS & 117.00 & 0.00 & 34.50 & 0.00 \\
ISIDA & 70.50 & 0.00 & 33.50 & 0.00 \\
SMSD & 245.50 & 0.00 & 98.00 & 0.00 \\
\hline
\end{tabular}

all); (2) the results up to or equal to the similarity thresholds $(0.90,0.80$ and 0.70$)$ are selected for each method; and (3) in each method, the threshold with the highest percentage of success in finding structures with the same activity is selected as the best threshold, and its results are compared.

As a result, a threshold of 0.90 was selected for the OBabel_FP2 method because 56\% of the 137 pairs of structures found presented the same activity (activeactive and inactive-inactive); for the remaining methods: SHAFTS, ISIDA, SMSD and MCPhd, thresholds of 0.80, $0.90,0.90$ and 0.70 were selected because they presented $53 \%, 55 \%, 65 \%$ and $67 \%$ of pairs of structures with the same activity respectively. Tables $8,9,10,11$ and 12 show the results that validate the selection.

If we analyze the results obtained with the best similarity thresholds in each method, we can infer that the percentage of structures found with the same activity (active-active and inactive-inactive) obtained with the MCPhd method (67\%) was better than the results with the OBabel_FP2, SHAFTS, ISIDA and SMSD methods by $11 \%, 14 \%, 12 \%$ and $2 \%$ respectively. Analyzing only the active-active pairs, the increase was 14\%, 16\%, 13\% and $11 \%$ ( $45 \%$ for MCPhd, 31\% for Obabel_FP2, 29\%

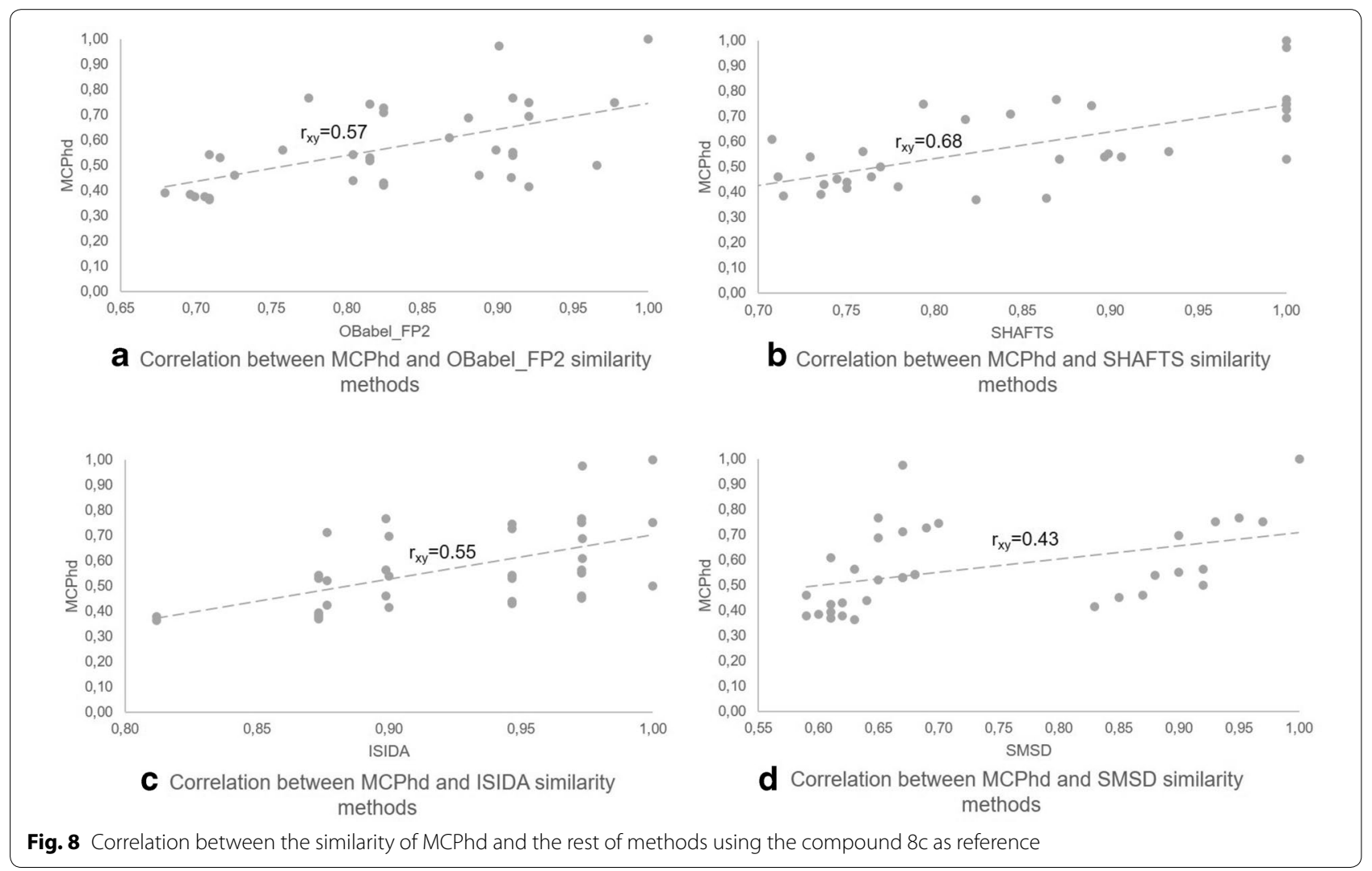




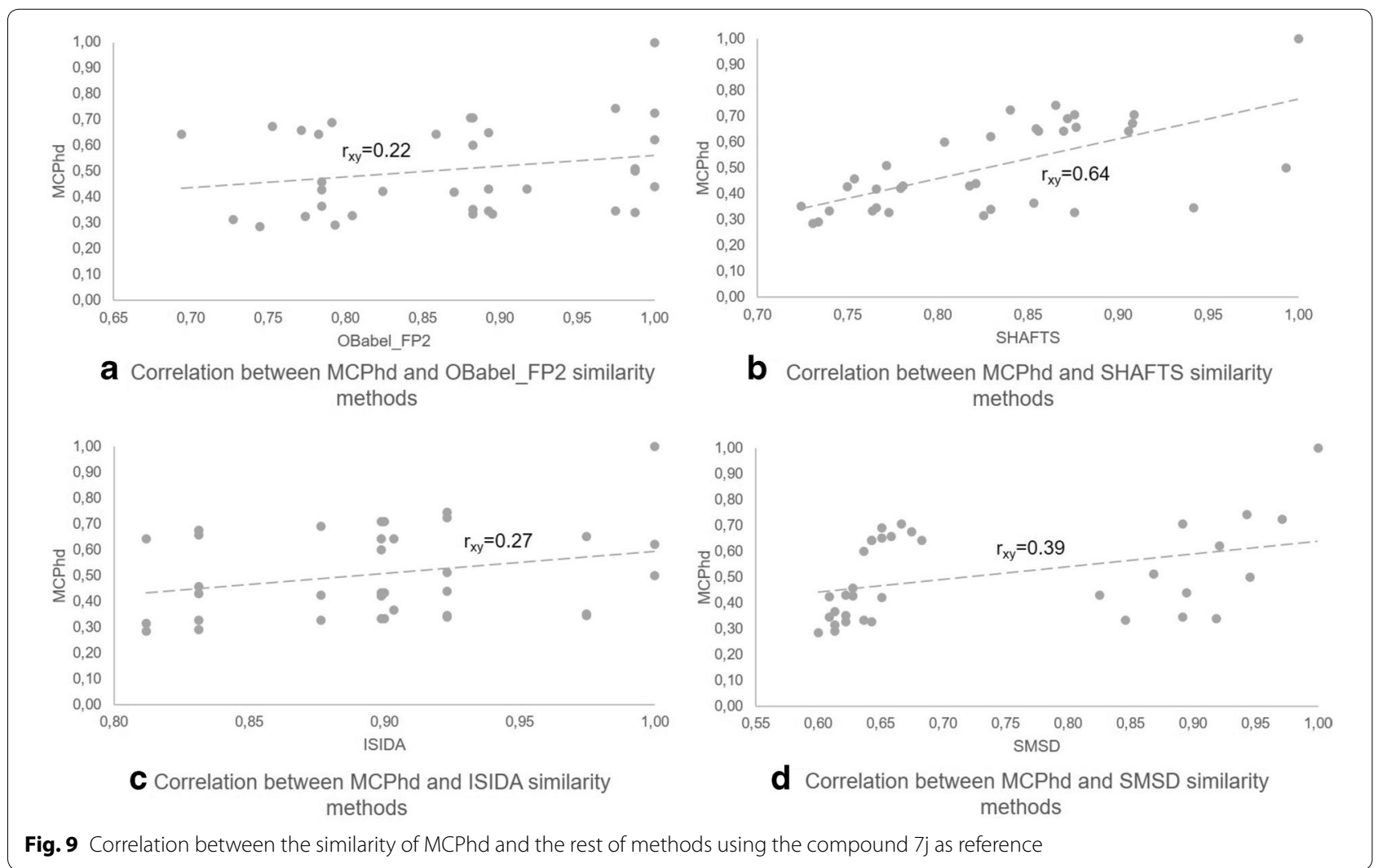

Table 6 Correlation results between MCPhd and the rest of methods for the active compounds in the dataset

\begin{tabular}{|c|c|c|c|c|c|}
\hline \multirow[t]{2}{*}{ Molecule } & \multirow[t]{2}{*}{$\mathrm{IC}_{50}$} & \multicolumn{4}{|c|}{ Correlation } \\
\hline & & $r_{x y} a$ & $r_{x y} b$ & $r_{x y} c$ & $r_{x y} d$ \\
\hline $8 c$ & 0.05 & 0.57 & 0.68 & 0.55 & 0.43 \\
\hline $7 c$ & 0.06 & 0.50 & 0.69 & 0.47 & 0.43 \\
\hline $8 f$ & 0.09 & 0.44 & 0.62 & 0.24 & 0.40 \\
\hline 81 & 0.09 & 0.45 & 0.71 & 0.33 & 0.53 \\
\hline $6 c$ & 0.106 & 0.45 & 0.50 & 0.40 & 0.44 \\
\hline $8 b$ & 0.12 & 0.49 & 0.05 & 0.36 & 0.35 \\
\hline 7| & 0.17 & 0.44 & 0.42 & 0.38 & 0.47 \\
\hline $6 i$ & 0.18 & 0.39 & 0.46 & 0.37 & 0.45 \\
\hline $8 i$ & 0.18 & 0.48 & 0.82 & 0.50 & 0.48 \\
\hline $8 d$ & 0.19 & 0.26 & 0.00 & 0.20 & 0.30 \\
\hline $8 e$ & 0.19 & 0.31 & 0.42 & 0.14 & 0.28 \\
\hline $8 \mathrm{~h}$ & 0.20 & 0.38 & 0.41 & 0.30 & 0.40 \\
\hline $8 a$ & 0.24 & 0.42 & 0.19 & 0.26 & 0.40 \\
\hline $7 f$ & 0.25 & 0.34 & 0.33 & 0.15 & 0.41 \\
\hline $7 b$ & 0.26 & 0.50 & -0.02 & 0.42 & 0.37 \\
\hline $7 i$ & 0.26 & 0.28 & 0.76 & 0.22 & 0.46 \\
\hline $7 \mathrm{~h}$ & 0.28 & 0.26 & 0.49 & 0.14 & 0.34 \\
\hline
\end{tabular}

a-OBabel_FP2 vs MCPhd, b-SHAFTS vs MCPhd,

c-ISIDA vs MCPhd and d-SMSD vs MCPhd for SHAFTS, 32\% for ISIDA and 34\% for SMSD). These results proved, once again, that the $\mathrm{MCPhd}$ method improved the similarity results obtained by the rest of similarity methods studied.

As another criterion, the 42 (OBabel_FP2), 75 (SHAFTS), 94 (ISIDA), 39 (SMSD) and 41 (MCPhd) pairs of compounds classified in the active-active category shown in Tables 8, 9, 10, 11, 12 were compared for all methods, using the best thresholds. To do so, only the 17 active compounds in the dataset were consided and, relationship graphs were drawn for the compounds present in the active-active pairs for each method. Figure 14 shows this representation.

If we observe the differences between the families of compounds 6, 7 and 8 (see Table 1), these differences were fundamentally due to the characteristics of the side chain. Moreover, the hybrid descriptors used in MCPhd have demonstrated $[35,45]$ their capability to distinguish between the same DC at different positions in a molecule. That allows MCPhd to find relationships/groups of compounds that show a higher functional relationship, and find similarities between compounds of different 


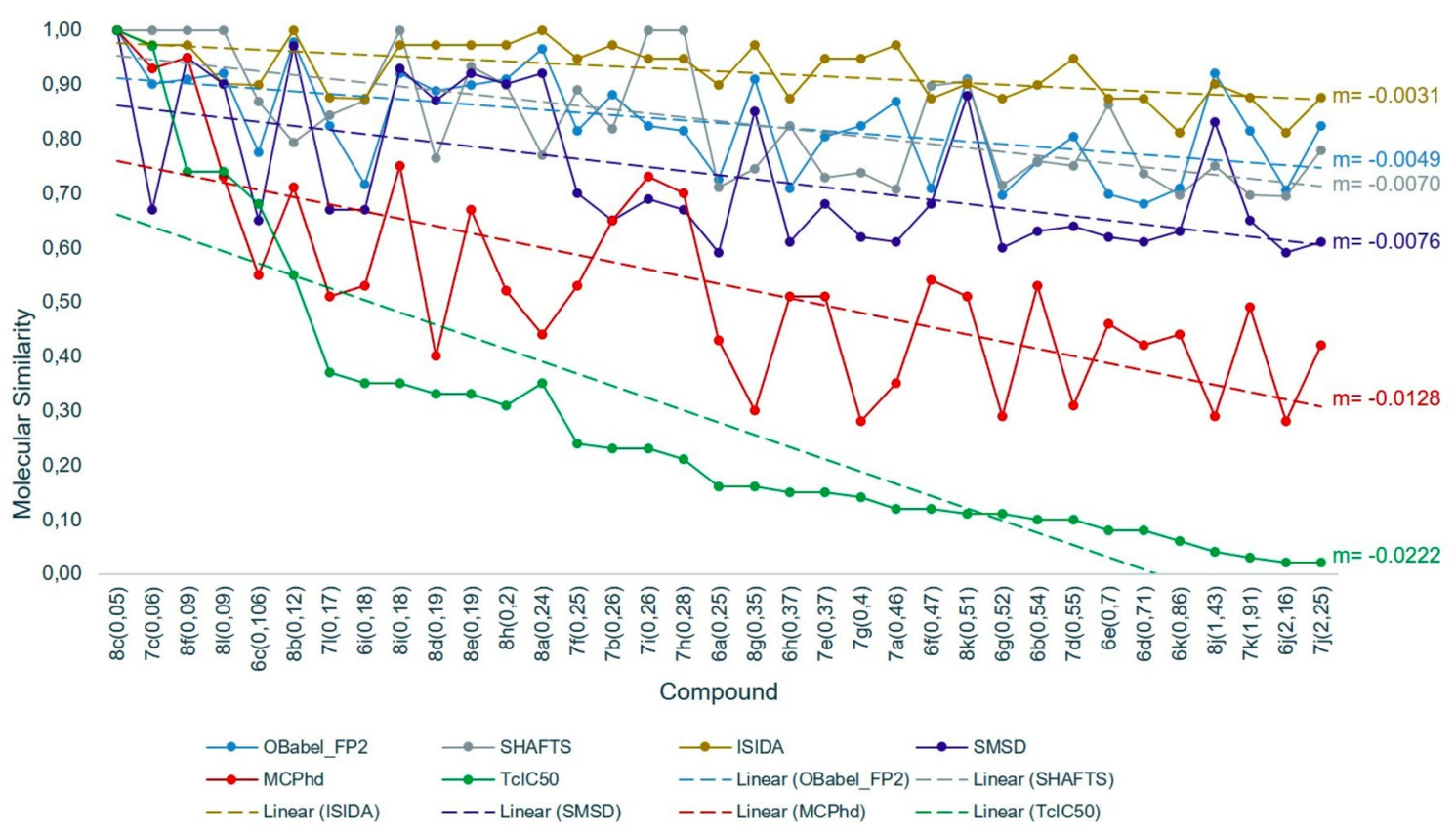

Fig. 10 Comparison of the molecular similarity between compound $8 \mathrm{c}$ and the rest of the dataset

families. The rest of the compared methods did not show this capacity. As shown in Fig. 14, whereas MCPhd did not relate compounds $8 \mathrm{~d}$ and $8 \mathrm{a}$ with the rest, suggesting that the electrostatic characteristics evidenced by the Electrotopographic State Index for Atoms was the source of the difference these two compounds. SMSD split this sample by grouping the three families separately, because it considers only structural features. SHAFTS considered that all compounds were related, and ISIDA identified similarity between $6 \mathrm{c}$ and $6 \mathrm{i}$ and the first of this pair with 8c.

This result implies that the MCPhd method allowed establishing similarity relations between compounds even from different families, making logical associations in contrast to the results of the other methods. The reason is that in addition to the structural information content provided by the electrotopographic state index for atoms, it includes the electrostatic information content.
As a last criterion, a runtime comparison was carried out for methods SMSD, SHAFTS and MCPhd. Figure 15 shows the box-plot representation of the computation time when calculating the similarity of each compound (reference structure) with the rest of the dataset. Moreover, the average runtime for SMSD was between 7688.2 and 8770.9 milliseconds, for SHAFTS was between 238.3 and 431.6 milliseconds. In contrast, the average calculation times for the MCPhd were between 18.6 and 35.6 milliseconds.

MCPhd uses a reduced graph, mapping smaller sized molecular graphs. In addition, the similarity calculated by the MCPhd method is based on the criterion of analogy or proximity between the physicochemical properties of the molecular fragments or subgroups that are compared by expressing these properties as a value of $S_{s t a t e}{ }_{3 D}$. On the other hand, SMSD and SHAFTS performs a more expensive mapping process for the compared molecular structures. 
Antelo-Collado et al. J Cheminform

(2020) $12: 61$

Page 14 of 22

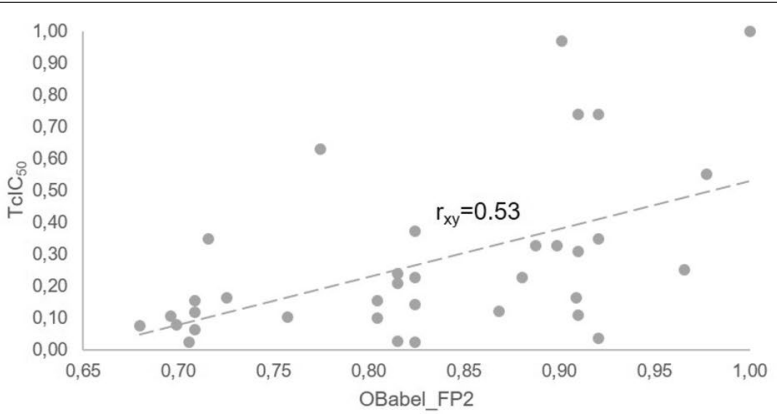

a Correlation between Obabel_FP2 similarity method and $\mathrm{TclC}_{50}$ similarity values.

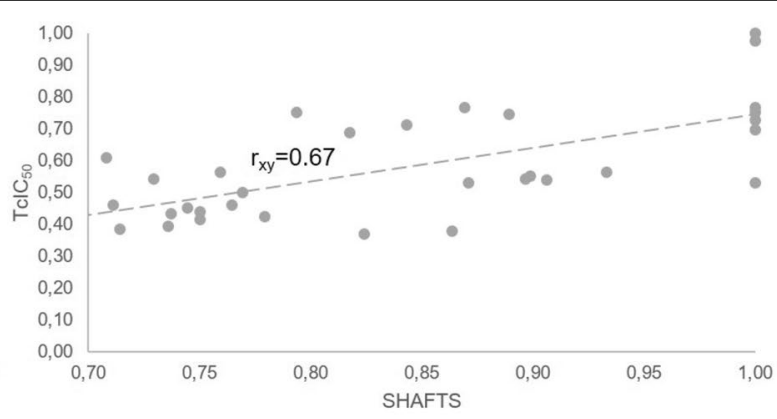

b Correlation between SHAFTS similarity method and $\mathrm{TcIC}_{50}$ similarity values.

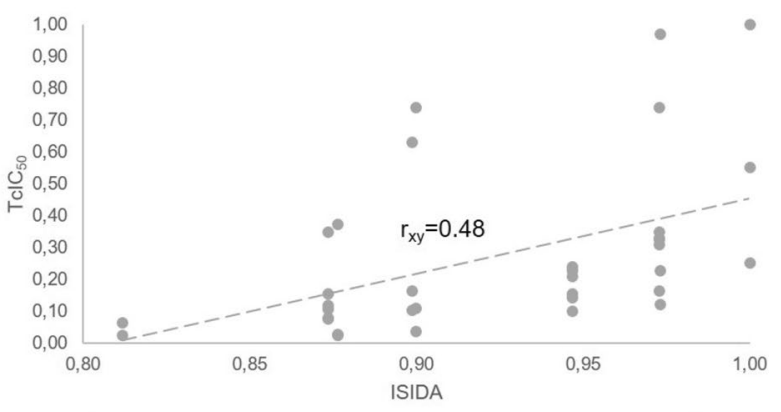

C Correlation between ISIDA similarity method and $\mathrm{TcIC}_{50}$ similarity values.

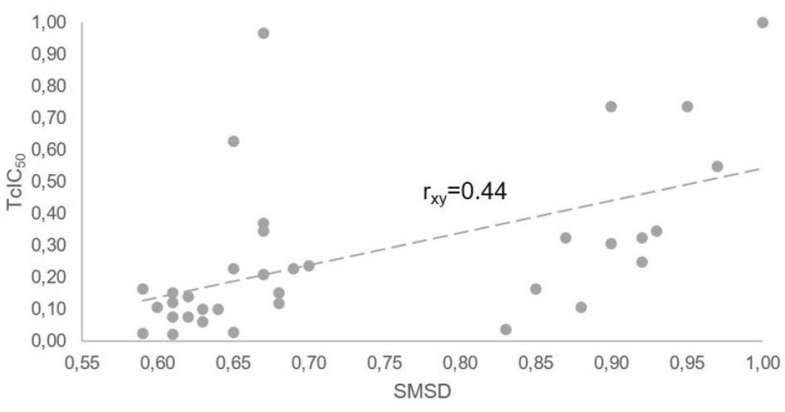

d Correlation between SMSD similarity method and $\mathrm{TCIC}_{50}$ similarity values.

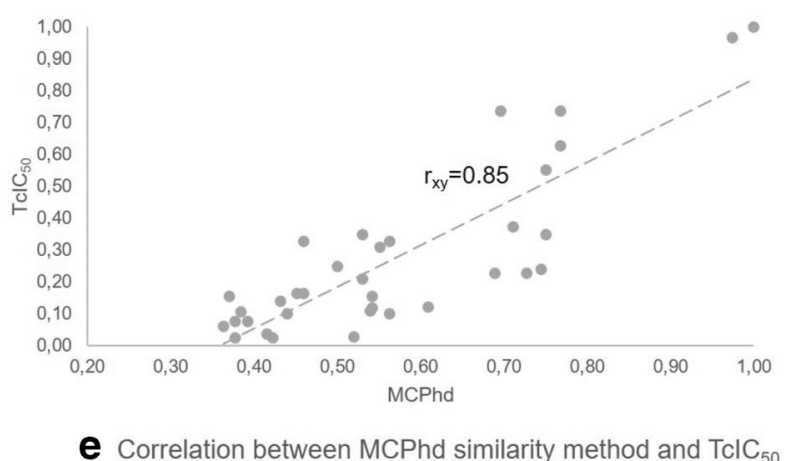
similarity values.

Fig. 11 Correlation between similarity methods and $T c C_{50}$ similarity values between compound $8 \mathrm{c}$ and the rest of the dataset 


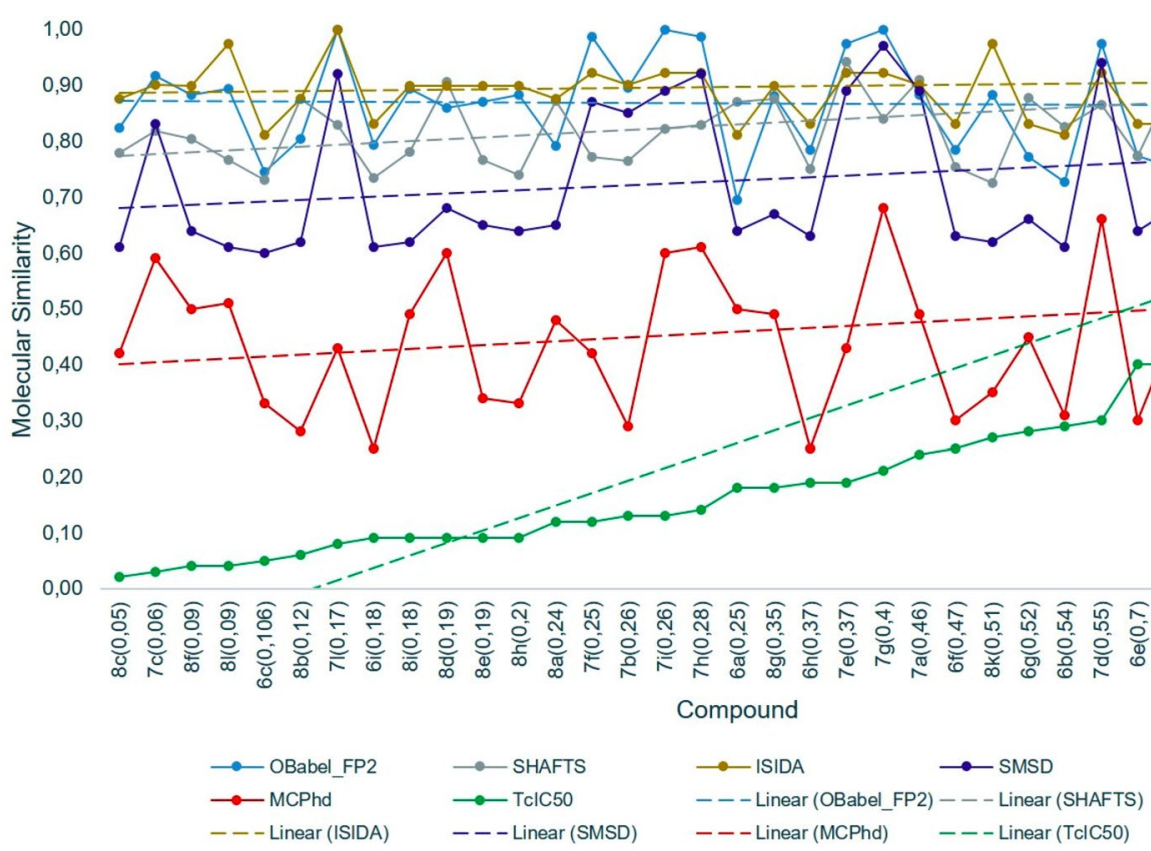

Fig. 12 Comparison of the molecular similarity between compound $7 j$ and the rest of the dataset

Table 7 Results of correlation between similarity methods and TcIC50 similarity values for the active compounds in the dataset

\begin{tabular}{|c|c|c|c|c|c|c|}
\hline \multirow[t]{2}{*}{ Molecule } & \multirow[t]{2}{*}{$\mathrm{IC}_{50}$} & \multicolumn{5}{|c|}{ Correlation } \\
\hline & & $r_{x y} a$ & $r_{x y} b$ & $r_{x y} c$ & $r_{x y} d$ & $r_{x y} e$ \\
\hline $8 c$ & 0.05 & 0.54 & 0.66 & 0.49 & 0.54 & 0.84 \\
\hline $7 c$ & 0.06 & 0.29 & 0.62 & 0.37 & 0.06 & 0.80 \\
\hline $8 f$ & 0.09 & 0.24 & 0.60 & 0.33 & 0.50 & 0.48 \\
\hline 81 & 0.09 & 0.26 & 0.62 & 0.04 & 0.44 & 0.55 \\
\hline $6 c$ & 0.106 & -0.11 & 0.10 & -0.03 & -0.13 & 0.64 \\
\hline $8 b$ & 0.12 & 0.50 & -0.06 & 0.49 & 0.45 & 0.44 \\
\hline 71 & 0.17 & 0.03 & 0.08 & -0.07 & -0.03 & 0.36 \\
\hline $6 i$ & 0.18 & -0.37 & 0.11 & -0.20 & -0.16 & 0.25 \\
\hline $8 i$ & 0.18 & 0.26 & 0.64 & 0.33 & 0.46 & 0.56 \\
\hline $8 d$ & 0.19 & 0.22 & -0.13 & 0.33 & 0.27 & -0.15 \\
\hline 8 e & 0.19 & 0.23 & 0.29 & 0.33 & 0.38 & 0.13 \\
\hline $8 \mathrm{~h}$ & 0.20 & 0.24 & 0.25 & 0.33 & 0.35 & 0.12 \\
\hline $8 a$ & 0.24 & 0.50 & -0.11 & 0.49 & 0.33 & -0.07 \\
\hline $7 f$ & 0.25 & -0.05 & 0.15 & 0.20 & 0.02 & 0.37 \\
\hline $7 b$ & 0.26 & 0.24 & -0.09 & 0.37 & -0.04 & 0.35 \\
\hline $7 i$ & 0.26 & -0.03 & 0.60 & 0.20 & -0.01 & 0.40 \\
\hline $7 \mathrm{~h}$ & 0.28 & -0.05 & 0.57 & 0.20 & -0.13 & -0.02 \\
\hline
\end{tabular}

a-OBabel_FP2 vs TcIC $C_{50}$, b-SHAFTS vs TcIC 50 , c-ISIDA vs TcIC ${ }_{50}$, d-SMSD vs $\mathrm{TCIC}_{50}$ and e-MCPhd vsTCIC 50 


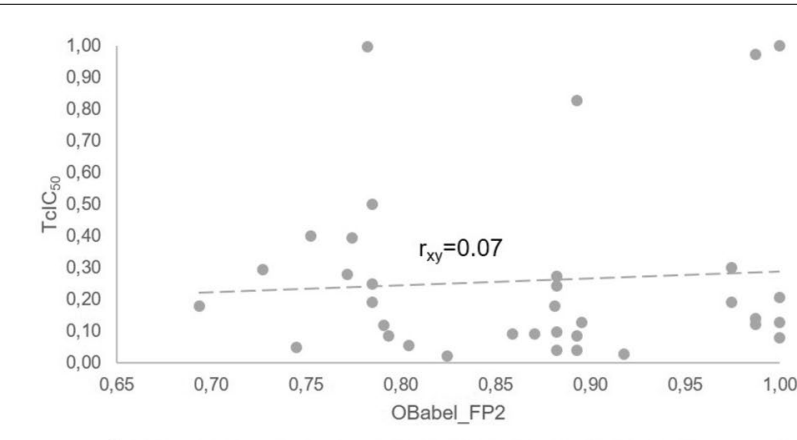

a Correlation between Obabel_FP2 similarity method and $\mathrm{TcIC}_{50}$ similarity values.

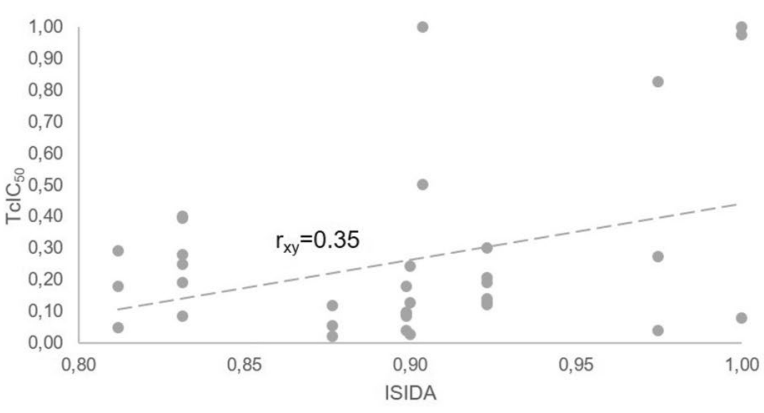

C Correlation between ISIDA similarity method and $\mathrm{TclC}_{50}$ similarity values.

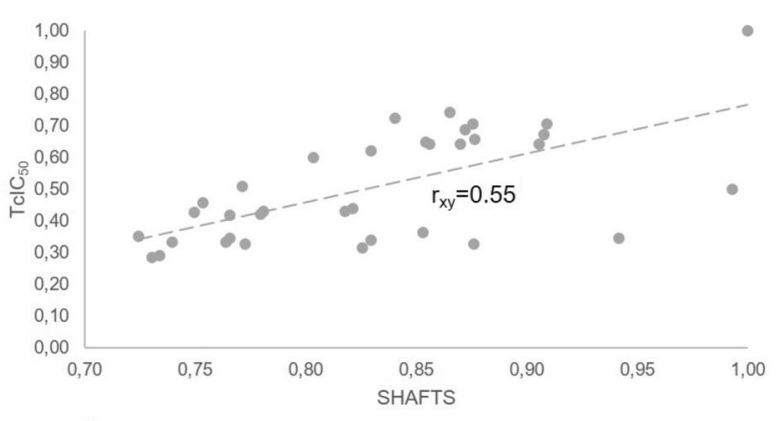

b Correlation between SHFTS similarity method and $\mathrm{TclC}_{50}$ similarity values.

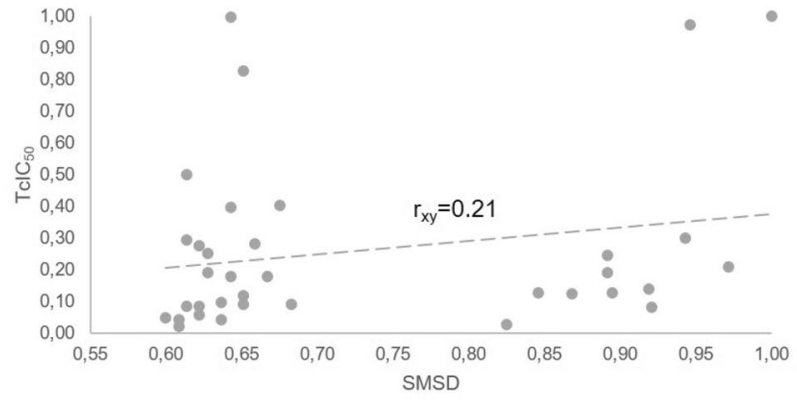

d Correlation between SMSD similarity method and $\mathrm{TclC}_{50}$ similarity values.

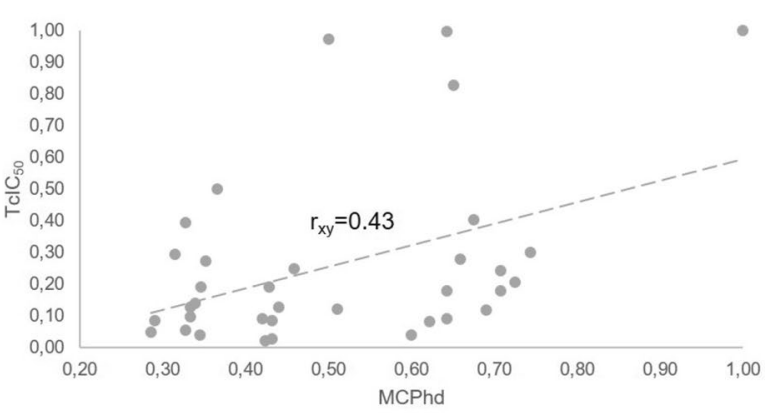

e Correlation between MCPhd similarity method and $\mathrm{TClC}_{50}$ similarity values.

Fig. 13 Correlation between similarity methods and $T c / C_{50}$ similarity values between compound $7 \mathrm{j}$ and the rest of the dataset 
Table 8 Comparison of the observed and predicted by OBabel_FP2 for several similarity thresholds

\begin{tabular}{|c|c|c|c|c|c|c|}
\hline Threshold & Real-Predicted & Pairs & $\%$ & Predicted & Pairs & $\%$ \\
\hline \multirow[t]{5}{*}{0.90} & Active-active & 42 & 31 & Correct & 77 & 56 \\
\hline & Inactive-inactive & 35 & 26 & & & \\
\hline & Active-inactive & 32 & 23 & Incorrect & 60 & 44 \\
\hline & Inactive-active & 28 & 20 & & & \\
\hline & Total & 137 & 100 & Total & 137 & 100 \\
\hline \multirow[t]{5}{*}{0.80} & Active-active & 101 & 32 & Correct & 170 & 55 \\
\hline & Inactive-inactive & 69 & 22 & & & \\
\hline & Active-inactive & 65 & 21 & Incorrect & 141 & 45 \\
\hline & Inactive-active & 76 & 24 & & & \\
\hline & Total & 311 & 100 & Total & 311 & 100 \\
\hline \multirow[t]{5}{*}{0.70} & Active-active & 133 & 24 & Correct & 277 & 49 \\
\hline & Inactive-inactive & 144 & 26 & & & \\
\hline & Active-inactive & 86 & 15 & Incorrect & 287 & 51 \\
\hline & Inactive-active & 201 & 36 & & & \\
\hline & Total & 564 & 100 & Total & 564 & 100 \\
\hline
\end{tabular}

Pairs-Number of predicted pairs

Table 9 Comparison of the observed and predicted by SHAFTS for several similarity thresholds

\begin{tabular}{|c|c|c|c|c|c|c|}
\hline Threshold & Real-predicted & Pairs & $\%$ & Predicted & Pairs & $\%$ \\
\hline \multirow[t]{5}{*}{0.90} & Active-active & 19 & 24 & Correct & 31 & 42 \\
\hline & Inactive-inactive & 13 & 18 & & & \\
\hline & Active-inactive & 18 & 24 & Incorrect & 43 & 58 \\
\hline & Inactive-active & 25 & 34 & & & \\
\hline & Total & 74 & 100 & Total & 74 & 100 \\
\hline \multirow[t]{5}{*}{0.80} & Active-active & 75 & 29 & Correct & 137 & 53 \\
\hline & Inactive-inactive & 62 & 24 & & & \\
\hline & Active-inactive & 43 & 17 & Incorrect & 120 & 47 \\
\hline & Inactive-active & 77 & 30 & & & \\
\hline & Total & 257 & 100 & Total & 257 & 100 \\
\hline \multirow[t]{5}{*}{0.70} & Active-active & 107 & 22 & Correct & 224 & 45 \\
\hline & Inactive-inactive & 117 & 24 & & & \\
\hline & Active-inactive & 76 & 15 & Incorrect & 271 & 55 \\
\hline & Inactive-active & 195 & 39 & & & \\
\hline & Total & 495 & 100 & Total & 495 & 100 \\
\hline
\end{tabular}

Pairs-Number of predicted pairs 
Table 10 Comparison of the observed and predicted by ISIDA for several similarity thresholds

\begin{tabular}{|c|c|c|c|c|c|c|}
\hline Threshold & Real-predicted & Pairs & $\%$ & Predicted & Pairs & $\%$ \\
\hline \multirow[t]{5}{*}{0.90} & Active-active & 94 & 32 & Correct & 165 & 55 \\
\hline & Inactive-inactive & 71 & 24 & & & \\
\hline & Active-inactive & 57 & 19 & Incorrect & 133 & 45 \\
\hline & Inactive-active & 76 & 26 & & & \\
\hline & Total & 298 & 100 & Total & 298 & 100 \\
\hline \multirow[t]{5}{*}{0.80} & Active-active & 136 & 23 & Correct & 289 & 49 \\
\hline & Inactive-inactive & 153 & 26 & & & \\
\hline & Active-inactive & 84 & 14 & Incorrect & 306 & 51 \\
\hline & Inactive-active & 222 & 37 & & & \\
\hline & Total & 595 & 100 & Total & 595 & 100 \\
\hline \multirow[t]{5}{*}{0.70} & Active-active & 136 & 23 & Correct & 289 & 49 \\
\hline & Inactive-inactive & 153 & 26 & & & \\
\hline & Active-inactive & 84 & 14 & Incorrect & 306 & 51 \\
\hline & Inactive-active & 222 & 37 & & & \\
\hline & Total & 595 & 100 & Total & 595 & 100 \\
\hline
\end{tabular}

Pairs-Number of predicted pairs

Table 11 Comparison of the observed and predicted by SMSD for several similarity thresholds

\begin{tabular}{|c|c|c|c|c|c|c|}
\hline Threshold & Real-predicted & Pairs & $\%$ & Predicted & Pairs & $\%$ \\
\hline \multirow[t]{5}{*}{0.90} & Active-active & 39 & 34 & Correct & 75 & 65 \\
\hline & Inactive-inactive & 36 & 31 & & & \\
\hline & Active-inactive & 19 & 17 & Incorrect & 41 & 35 \\
\hline & Inactive-active & 22 & 19 & & & \\
\hline & Total & 116 & 100 & Total & 116 & 100 \\
\hline \multirow[t]{5}{*}{0.80} & Active-active & 52 & 28 & Correct & 106 & 57 \\
\hline & Inactive-inactive & 54 & 29 & & & \\
\hline & Active-inactive & 48 & 26 & Incorrect & 81 & 43 \\
\hline & Inactive-active & 33 & 18 & & & \\
\hline & Total & 187 & 100 & Total & 187 & 100 \\
\hline \multirow[t]{5}{*}{0.70} & Active-active & 63 & 30 & Correct & 121 & 57 \\
\hline & Inactive-inactive & 58 & 27 & & & \\
\hline & Active-inactive & 48 & 23 & Incorrect & 91 & 43 \\
\hline & Inactive-active & 43 & 20 & & & \\
\hline & Total & 212 & 100 & Total & 212 & 100 \\
\hline
\end{tabular}

Pairs-Number of predicted pairs 
Table 12 Comparison of the observed and predicted by MCPhd for several similarity thresholds

\begin{tabular}{|c|c|c|c|c|c|c|}
\hline Threshold & Real-predicted & Pairs & $\%$ & Predicted & Pairs & $\%$ \\
\hline \multirow[t]{5}{*}{0.90} & Active-active & 14 & 33 & Correct & 26 & 62 \\
\hline & Inactive-inactive & 12 & 29 & & & \\
\hline & Active-inactive & 8 & 19 & Incorrect & 16 & 38 \\
\hline & Inactive-active & 8 & 19 & & & \\
\hline & Total & 42 & 100 & Total & 42 & 100 \\
\hline \multirow[t]{5}{*}{0.80} & Active-active & 16 & 36 & Correct & 28 & 62 \\
\hline & Inactive-inactive & 12 & 27 & & & \\
\hline & Active-inactive & 9 & 20 & Incorrect & 17 & 38 \\
\hline & Inactive-active & 8 & 18 & & & \\
\hline & Total & 45 & 100 & Total & 45 & 100 \\
\hline \multirow[t]{5}{*}{0.70} & Active-active & 41 & 45 & Correct & 62 & 67 \\
\hline & Inactive-inactive & 23 & 25 & & & \\
\hline & Active-inactive & 15 & 16 & Incorrect & 30 & 33 \\
\hline & Inactive-active & 15 & 16 & & & \\
\hline & Total & 92 & 100 & Total & 92 & 100 \\
\hline
\end{tabular}

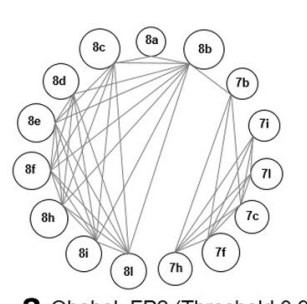

a Obabel_FP2 (Threshold 0.90)

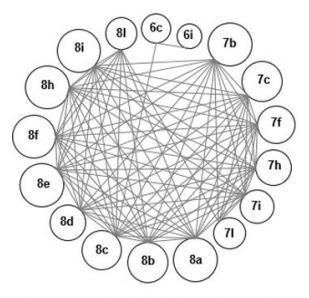

C ISIDA (Threshold 0.90)

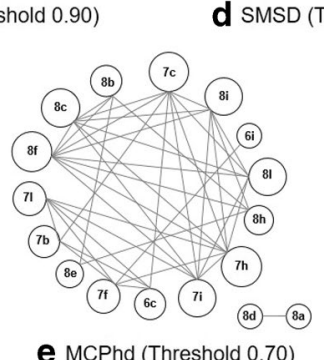

Fig. 14 Relationship graph of active compounds with a molecular similarity higher than the selected threshold
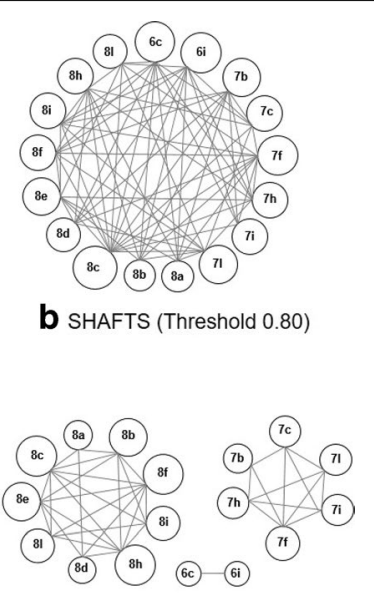

SMSD (Threshold 0.90)

\section{Conclusions}

This work proposed a new approach that uses the 3D structure of molecules with physicochemical information to estimate the molecular similarity between chemical compounds. The method has been favorably compared with the standard SMSD, OBabel_FP2, ISIDA and SHAFTS methods and shows better performance in obtaining structures with the same activity using similarity cutoff values during the screening process. Furthermore, the proposal shows the ability to find similar compounds among different families. This strongly suggest the possibility of employing the MCPhd method for isosteric studies.

Finally, the proposal presented in this paper provides a promising method for extending this method to be used in the construction of QSAR models for molecular activity prediction.

\section{Supplementary information}

Supplementary information accompanies this paper at https://doi. org/10.1186/s13321-020-00462-3.

Additional file 1. rar-file containing all necessary data/files to reproduce the results presented in this work.

\section{Acknowledgements}

Not applicable. 


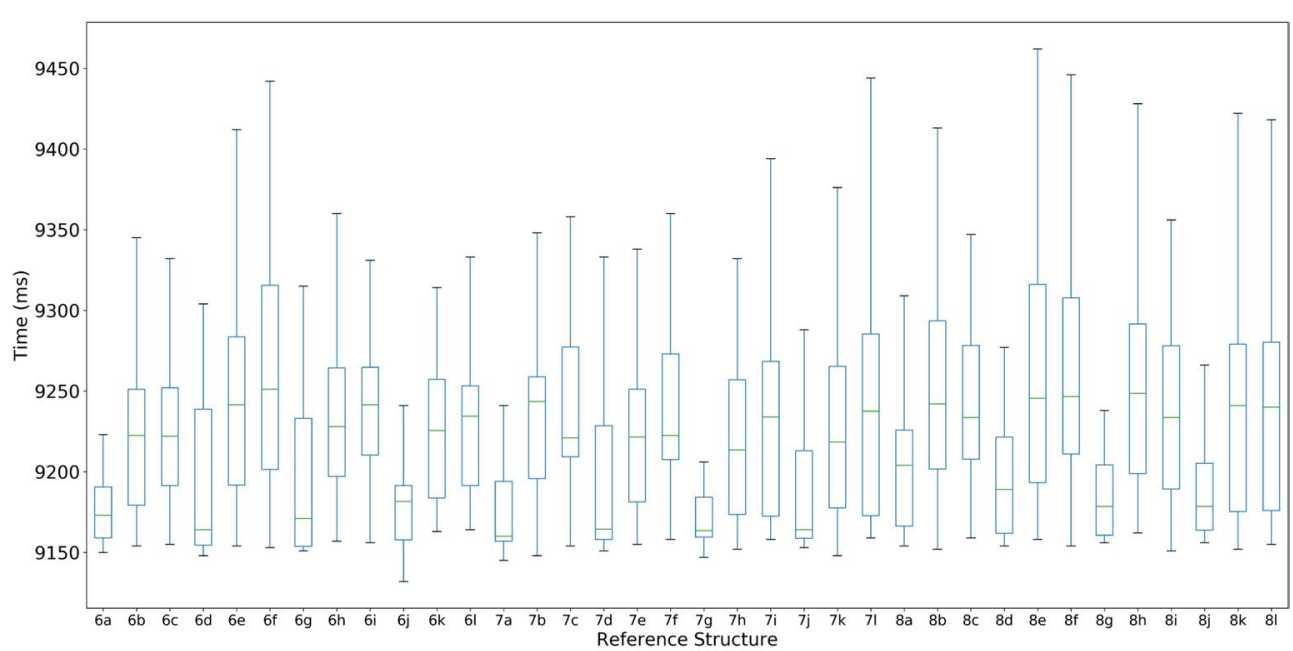

a SMSD

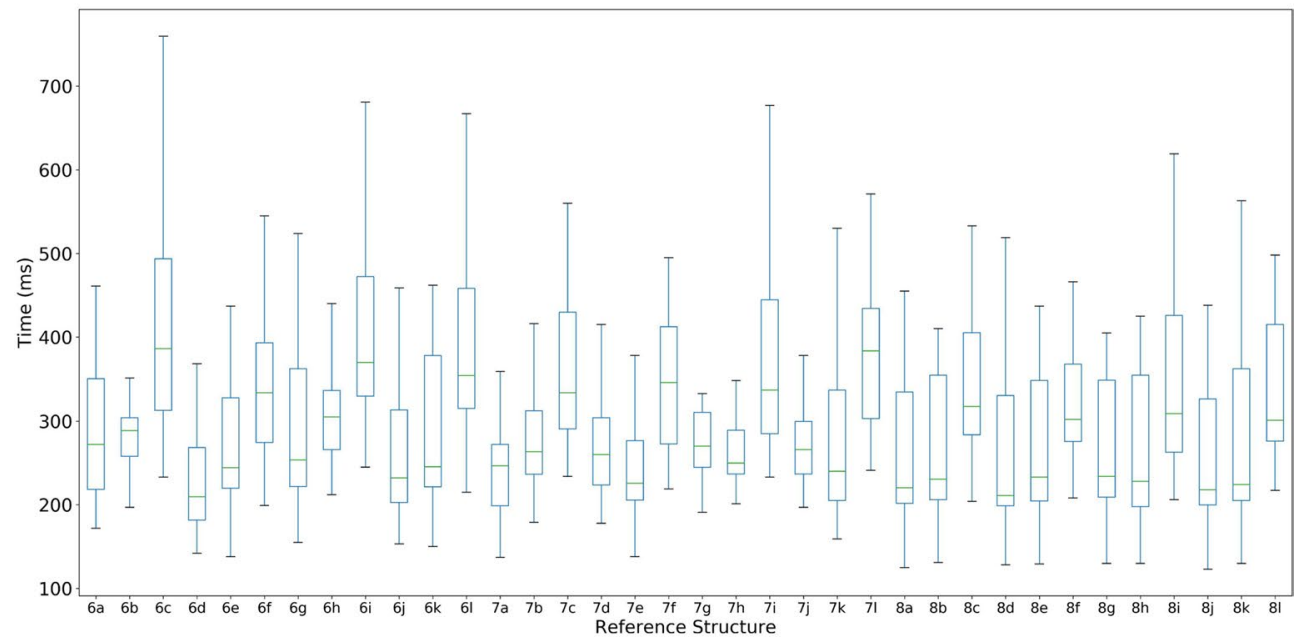

b SHAFTS

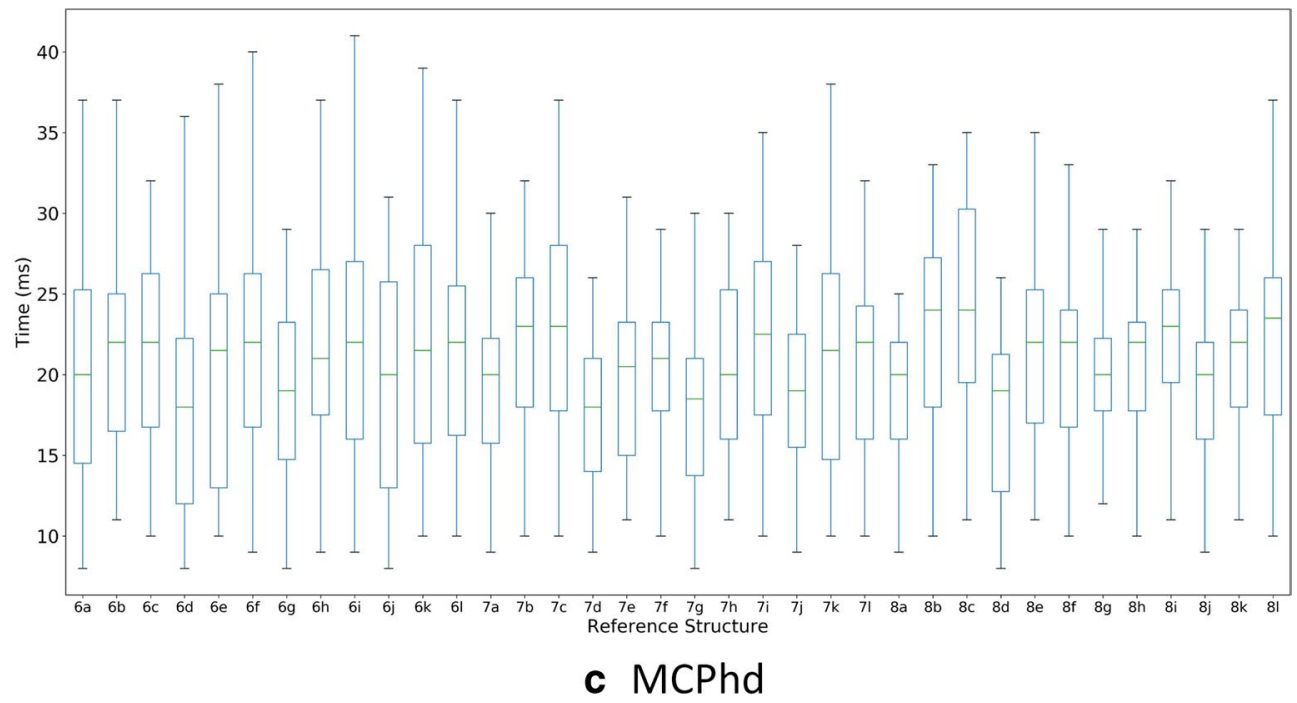

Fig. 15 Calculation times 


\section{Authors' contributions}

Authors contributed equally to this work. All authors read and approved the final manuscript.

\section{Funding}

This work was supported in part by Project PID2019-109481GB-100 of the Spanish Ministry of Science and Innovation, by Project 1264182-F of the Andalusian Regional Government, by Project PP2019-Submod-1.2 of the Cordoba University and by Project AC25_2017 of the University of Informatics Sciences.

\section{Availability of data and materials}

All the data on which the conclusions of the work are based have been exhaustively presented in the manuscript. The algorithm implementation for free use, the dataset used in the paper, and other files needed to reproduce the results are included as supplementary material. The source code under GNU General Public License v3.0 can be downloaded from the GitHub repository at the following link: https://github.com/aantelo00/MCPhd.

\section{Competing interests}

The authors declare that they have no competing interests.

\section{Author details}

${ }^{1}$ University of Informatics Science, Carretera San Antonio de los Baños Km. 2 1/2, Boyeros, La Habana, Cuba, Havana, Cuba. ${ }^{2}$ Department of Computing and Numerical Analysis, University of Cordoba, Campus de Rabanales, Albert Einstein Building, E-14071 Córdoba, Spain.

Received: 17 April 2020 Accepted: 14 September 2020

Published online: 09 October 2020

\section{References}

1. Maggiora G, Vogt M, Stumpfe D, Bajorath J (2013) Molecular similarity in medicinal chemistry. J Med Chem 57:3186-3204. https://doi. org/10.1021/jm401411z

2. Kunimoto R, Vogt M, Bajorath J (2016) Maximum common substructurebased Tversky index: an asymmetric hybrid similarity measure. J Comput Aided Mol Des 30:523-531. https://doi.org/10.1007/s10822-016-9935-y

3. Vogt M, Stumpfe D, Geppert H, Bajorath J (2010) Scaffold hopping using two-dimensional fingerprints: true potential, black magic, or a hopeless endeavor? guidelines for virtual screening. J Med Chem 12:5707-5715. https://doi.org/10.1021/jm100492z

4. Gardiner EJ, Holliday JD, O'dowd C, Willett P (2011) Effectiveness of 2D fingerprints for Scaffold hopping. Future Med Chem 3:405-414. https:// doi.org/10.4155/fmc.11.4

5. Good AC, Richards WG (1998) Explicit calculation of 3D molecular similarity. Perspect Drug Discovery Des. 9:321-338. https://doi. org/10.1023/A:1027280526177

6. Rush TS, Grant JA, Mosyak L, Nicholls A (2005) A shape-based 3-D Scaffold hopping method and its application to a bacterial protein-protein interaction. J Med Chem. 48:1489-1495. https://doi.org/10.1021/jm0401630

7. Moffat K, Gillet VJ, Whittle M, Bravi G, Leach AR (2008) A comparison of field-based similarity searching methods: CatShape, FBSS, and ROCS. J Chem Inf Model 48:719-729. https://doi.org/10.1021/ci700130j

8. Tresadern G, Bemporad D (2010) Modeling approaches for ligand-based 3D similarity. Future Med Chem. 2:1547-1561. https://doi.org/10.4155/ fmc. 10.244

9. Hu Y, Stumpfe D, Bajorath J (2017) Recent advances in scaffold hopping. J Med Chem 60:1238-1246. https://doi.org/10.1021/acs.jmedchem.6b014 37

10. Kenny PW, Sadowski J (2005) Structure modification in chemical databases. Methods and Principles in Medicinal Chemistry. Wiley-Vch, Weinheim 23:271-285. https://doi.org/10.1002/3527603743.ch11

11. Hussain J, Rea C (2010) Computationally efficient algorithm to identify matched molecular Pairs (Mmps) in large data sets. J Chem Inf Model 50:339-348. https://doi.org/10.1021/ci900450m

12. Duesbury E, Holliday JD, Willett $P$ (2017) Maximum common subgraph isomorphism algorithms. Match Commun Math Comput Chem 77:213-232
13. Cerruela García G, Luque Ruiz I, Gómez-Nieto MÁ (2004) Step-by-Step calculation of all maximum common substructures through a constraint satisfaction based algorithm. J Chem Informat Comput Sci 44:30-41. https://doi.org/10.1021/ci034167y

14. Cerruela García G, Palacios-Bejarano B, Luque Ruiz I, Gómez-Nieto MÁ (2012) Comparison of representational spaces based on structural information in the development of QSAR models for benzylamino enaminone derivatives. SAR QSAR Environ Res 23(7-8):751-774

15. Maggiora GM, Shanmugasundaram V (2004) Molecular similarity measures. Methods Mol Biol 275:1-50. https://doi.org/10.1385/1-59259 $-802-1: 001$

16. Zhang B, Vogt M, Maggiora GM, Bajorath J (2015) Design of chemical space networks using a tanimoto similarity variant based upon maximum common substructures. J Comput Aided Mol Des 29:937-950. https:// doi.org/10.1007/s10822-015-9872-1

17. Carrasco-Velar R (2007) Nuevos descriptores atómicos y moleculares para estudios de estructura-actividad: Aplicaciones. Editorial Universitaria, Ciudad de La Habana: 1-141. ISBN 978-959-16-0646-4

18. Kier LB, Hall LH (1990) An electrotopological-State index for atoms in molecules. Pharm Res 7:801-807. https://doi.org/10.1023/A:1015952613 760

19. Nikolova N, Jaworska J (2003) Approaches to measure chemical similaritya review. QSAR Combinat Sci 22(9-10):1006-1026

20. Raymond JW, Willett $P$ (2003) Similarity searching in databases of flexible 3D structures using smoothed bounded distance matrices. J Chem Inform Comput Sci 43(3):908-916

21. Crippen GM, Havel TF (1988) Distance geometry and molecular conformation, vol 74. Research Studies Press, Taunton

22. Hu J, Liu Z, Yu DJ, Zhang Y (2018) LS-align: an atom-level, flexible ligand structural alignment algorithm for high-throughput virtual screening. Bioinformatics 34(13):2209-2218

23. Liu X, Jiang H, Li H (2011) SHAFTS: a hybrid approach for 3D molecular similarity calculation. 1. Method and assessment of virtual screening. J Chem Inform Model 51(9):2372-2385

24. Quintus F, Sperandio O, Grynberg J, Petitjean M, Tuffery P (2009) Ligand scaffold hopping combining 3D maximal substructure search and molecular similarity. BMC Bioinformatics 10(1):1-11

25. Kumar A, Zhang KY (2018) Advances in the development of shape similarity methods and their application in drug discovery. Front Chem 6:315

26. O'Boyle NM, Banck M, James CA, Morley C, Vandermeersch T, Hutchison GR (2011) Open babel: an open chemical toolbox. J Cheminform 3(1):33

27. CoSiAn (Combinatorial Similarity Analysis) Webserver (2020). http://cosia n.cbs.cnrs.fr/from.html. Accessed 28 Jul 2020

28. Jiayu G, Chaoqian C, Xiaofeng L, Xin K, Hualiang J, Daqi G, Honglin L (2013) ChemMapper: a Versatile web server for exploring pharmacology and chemical structure association based on molecular 3D similarity method. Bioinformatics. 29:1827-1829. https://doi.org/10.1093/bioin formatics/btt270

29. Rahman SA, Bashton M, Holliday GL, Schrader R, Thornton JM (2009) Small molecule subgraph detector (SMSD) Toolkit. J Cheminform 1:12. https://doi.org/10.1186/1758-2946-1-12

30. Fast 3D Structure Generation with CORINA Classic (2020). https://www. mn-am.com/online demos/corina_demo. Accessed 18 Feb 2020

31. Varnek A, Fourches D, Horvath D, Klimchuk O, Gaudin C, Vayer P, Marcou G (2008) ISIDA-Platform for virtual screening based on fragment and pharmacophoric descriptors. Current Computer-Aided Drug Design 4(3):191

32. Software Solutions and Services for Chemistry \& Biology (2020). http:// www.chemaxon.com. Accessed 30 Jul 2020

33. Willighagen EL, Mayfield JW, Alvarsson J et al (2017) The chemistry development kit (CDK)V2.0: atom typing, depiction, molecular formulas, and substructure searching. J Cheminf 9:33. https://doi.org/10.1186/s1332 1-017-022

34. Weis R, Seebacher W, Brun R, Kaiser M, Sat R, Faist J (2013) 4-Aminobicyclo[2.2.2]octan-2-yl 4-aminobutanoates with antiprotozoal activity. Monatsh Chem. https://doi.org/10.1007/s00706-013-1116-2

35. Carrasco R, Prieto JO, Antelo A, Padrón JA, Cerruela G, Maceo ÁL, Alcolea R, Silva LG (2013) Hybrid reduced graph For SAR studies. SAR QSAR Environ Res 24:201-214. https://doi.org/10.1080/1062936X.2013.764926 
36. Avidon W, Pomerantsev IA, Golender VE, Rozenblit AB (1982) Structureactivity relationship oriented languages for chemical structure representation. J Chem Inf Comp Sci 22:207-214

37. Steffen A, Kogej T, Tyrchan C, Engkvist O (2009) Comparison of molecular fingerprint methods on the basis of biological profile data. J Chem Inform Model 49(2):338-347

38. Lance GN, Williams WT (1966) Computer programs for hierarchical polythetic classification ("similarity analysis"). Comput J 9:60-64

39. Friedman HL (1951) Influence of isosteric replacements upon biological activity. Nat Acad Sci Nat Res Council 206:295

40. Burger A (1991) Isosterism and bioisosterism in drug design in Progress in Drug Research. 37:287-371. https://doi.org/10.1007/978-3-0348-7139-6_7

41. Lassalas P, Oukoloff K, Makani V, James M, Tran V, Yao Y, Huang L, Vijayendran K, Monti L, Trojanowski JQ, Lee VM, Kozlowski MC, Smith AB III, Brunden KR, Ballatore C (2017) Evaluation of Oxetan-3-ol, Thietan-3-ol, and derivatives thereof as bioisosteres of the carboxylic acid functional group. ACS Med Chem. 8:864-868. https://doi.org/10.1021/acsmedchem lett.7b00212
42. Tahirova N, Poivet E, Xu L, Peterlin Z, Zou DJ, Firestein SS (2019) Bioisosterism reveals new structure-odor relationships. bioRxiv. https://doi. org/10.1101/567701

43. Mann HB, Whitney DR (1947) On a test of whether one of two random variables is stochastically larger than the other. Ann Mathe Stat 18:50-60. https://doi.org/10.1214/aoms/1177730491

44. Baptista I, Camila Otero C, González S, Pertegás A, Galvez J, García R (2019) Aplicación de la topología molecular al análisis de la actividad antimalárica de 4-Aminobiciclo [2.2.2]Octan-2 il 4-Aminobutanoatos y sus análogos etanoatos y propanoatos. Nereis 11:51-65

45. Antelo A, Paneque JL, Hernández MC, Ramón Carrasco R (2016) Molecular similarity using hybrid indices. Cuban J Med Inform 8:487-498

\section{Publisher's Note}

Springer Nature remains neutral with regard to jurisdictional claims in published maps and institutional affiliations.
Ready to submit your research? Choose BMC and benefit from:

- fast, convenient online submission

- thorough peer review by experienced researchers in your field

- rapid publication on acceptance

- support for research data, including large and complex data types

- gold Open Access which fosters wider collaboration and increased citations

- maximum visibility for your research: over $100 \mathrm{M}$ website views per year

At BMC, research is always in progress.

Learn more biomedcentral.com/submissions 